\title{
1 Delayed aneuploidy stress response of neural stem cells impairs adult
}

\section{2 lifespan in flies}

3

4 Mihailo Mirkovic*, Leonardo G. Guilgur*\#, Diogo Passagem-Santos, Raquel A. Oliveira

5

6 Instituto Gulbenkian de Ciência, Rua Quinta Grande 6, Oeiras, Portugal 2780-156

7

$8 \quad *$ These authors contributed equally

9 \# Correspondence should be addressed to LGG (lguilgur@igc.gulbenkian.pt) and RAO

10 (rcoliveira@igc.gulbenkian.pt)

11

12 ORCID id: Mihailo Mirkovic (orcid.org/ 0000-0003-0802-7200)

13 ORCID id: Leonardo G. Guilgur (orcid.org/ 0000-0002-8197-5920)

14 ORCID id: Raquel A. Oliveira (orcid.org/0000-0002-8293-8603) 


\section{Summary (150)}

16 Studying aneuploidy during organism development has strong limitations, as chronic mitotic

17 perturbations used to generate aneuploidy result in lethality. We developed a genetic tool to 18 induce aneuploidy in an acute and time controlled manner during Drosophila development. This

19 is achieved by reversible depletion of cohesin, a key molecule controlling mitotic fidelity.

20 Larvae challenged with aneuploidy hatch into adults with severe motor defects shortening their 21 lifespan. Neural stem cells, despite being aneuploid, display a delayed stress response and 22 continue proliferating, resulting in the rapid appearance of chromosomal instability, complex 23 array of karyotypes and cellular abnormalities. Notably, when other brain cell-lineages are 24 forced to self-renew, aneuploidy-associated stress response is significantly delayed, indicating 25 that stemness state confers resistance to aneuploidy. Sparing solely the developing brain from 26 induced aneuploidy is sufficient to rescue motor defects and adult lifespan, suggesting that neural tissue is the most ill-equipped to deal with developmental aneuploidy.

\section{Keywords}

30 Cohesin, Aneuploidy, Neural Stem Cells, Drosophila

\section{Highlights}

- Reversible depletion of cohesin results in just a round or two of aberrant cell divisions, generating high levels of aneuploidy.

- Larvae challenged with aneuploidy during development hatch into impaired adults.

- Few cell cycles are sufficient for chromosomal instability to emerge from a previously stable aneuploid state.

- Neural stemness delays aneuploidy stress response. 


\section{Introduction}

Aneuploidy, a state of chromosome imbalance, was observed over a century ago by Theodor Boveri. Since then, numerous studies have shown that aneuploidy is largely detrimental both at cellular and organism level. In multicellular organisms chromosome gain or loss results in lethality or developmental defects $(1,2)$. At the cellular level, studies in yeast and cell culture have demonstrated that aneuploidy has a high fitness cost for the cell, as unbalanced karyotypes lead to activation of multiple stress response pathways, resulting in reduced proliferation, cell cycle arrest, or cell death (Reviewed in (3). The aneuploidy stress response and consequential drop in fitness seems at odds with the hypothesized role of aneuploidy in promoting malignancy, which is usually marked by over-proliferation (4). Ninety percent of solid tumors harbor whole chromosome gains and/or losses (5). Therefore, although usually detrimental to cell fitness, aneuploidy and its effects on cell proliferation can be context dependent, which emphasizes our need for a better understanding of the immediate and ultimate consequences of this abnormal cellular condition in a tissue context and through development.

Study of aneuploidy in vivo is challenging since somatic aneuploidy is a rare event and is difficult to capture and trace in real time due to several constraints: i) Cells are equipped with surveillance mechanisms that prevent chromosome mis-segregation (e.g Spindle Assembly Checkpoint (SAC) (Reviewed in (6) making naturally occurring aneuploidy events virtually impossible to evaluate; ii) experimentally-induced aneuploidy, by compromising mitotic fidelity, if often of low prevalence, as it has been demonstrated for several mammalian (7) (8) and Drosophila tissues $(9,10)$ and iii) induction of somatic or constitutional aneuploidy in metazoans relies on chronic mitotic perturbation (Listed in (11) which usually causes embryonic lethality (Reviewed in(12) as a result of progressive accumulation of damage in the developing organism. Thus, from these studies, it is impossible to disentangle short term and long term consequences of aneuploidy, or to examine kinetics of the response to aneuploid state during development. To circumvent these limitations, we generated a genetic system with the power to induce aneuploidy in an acute and time-controlled manner, in all the dividing tissues of the developing Drosophila. The tool is based on reversible depletion of cohesin, a key molecule regulating mitotic fidelity $(13,14)$. Cohesin is a tripartite ring complex, composed by SMC1, SMC3 and the bridging kleisin subunit $\operatorname{RAD} 21(15,16)$. The primary mitotic role of cohesin is to mediate sister chromatid cohesion, by topologically entrapping DNA fibers from neighboring chromatids $(17,18)$. Cells entering mitosis with premature loss of cohesion and sister chromatid separation activate the Spindle Assembly Checkpoint (SAC) resulting in prolonged mitosis (14, 19). During this SAC-dependent mitotic delay, chromosomes are shuffled from one cell pole to 
randomization and aneuploidy upon mitotic exit with a theoretical rate of nearly $100 \%$. Our engineered system enables a quick restoration of this complex shortly after its inactivation, thereby restricting mitotic abnormalities to a short time-frame, concomitantly with the generation of high levels of aneuploidy. Using such tool, we dissect the kinetics of aneuploidy response across various cell/tissue types and developmental timings.

\section{Results}

A genetic system for acute and time-controlled generation of aneuploidy in a developing organism

To induce aneuploidy in an acute and time-controlled manner, we developed a genetic system based on rapid removal of cohesin complex, the molecular glue that holds sister chromatids together. To prevent a chronic cohesion depletion state and restrict mitotic failure to a single cell cycle, our genetic system is able to induce cohesin inactivation, followed by subsequent cohesion rescue. The system relies on the artificial cleavage of a modified version of the RAD21 cohesin subunit that contains TEV protease cleavage sites (RAD21-TEV). As previously shown, this system is very efficient at inactivating cohesin upon expression of the exogenous Tobacco Etch Virus (TEV) protease, induced by a heat-shock promoter, resulting in long-term inactivation of this complex ( $>24 \mathrm{~h}$ ) (20). To restrict cohesin impairment, we modified this system by promptly rescuing cohesin integrity through the expression of TEV-resistant RAD21 protein (RAD21-WT) right after TEV-mediated inactivation. For this purpose, RAD21WT expression is under the control of UAS promoter (UAS-Rad21-wt-myc) that is induced by a Gal4 protein induced concomitantly with the TEV protease (also under a heat-shock promoter, HSprom-GAL4) (Figure 1A). Given that the TEV protease is under a direct control of heatshock promoter, whereas RAD21-WT relies on a dual expression-system (Gal4-UAS), we anticipated that the temporal delay in RAD21-WT expression relative to the induction of TEV

103 protease would lead to a short time window of cohesin inactivation (RAD21 cleavage) (Figure 104 1A).

105 To test this, we probed for the kinetics of TEV-mediated cleavage of RAD21-TEV and 106 synthesis of RAD21-WT in different tissues of the developing larvae. After heat shock, both

107 Drosophila larvae brains and wing discs, showed similar kinetics of the TEV-sensitive RAD21

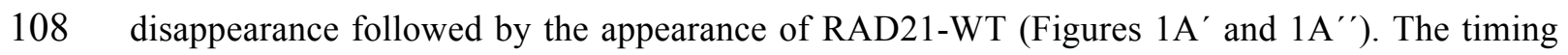
109 of protein depletion/re-establishment differs slightly among different tissues or developmental 110 stages, but leads on average to a period of $\sim 1$ hour without cohesin (Figures $1 \mathrm{~A}^{\prime}$; $1 \mathrm{~A}^{\prime \prime}$ and 111 S1B). 
113 Reversible removal of cohesin results in a single round of mitotic abnormalities and

\section{4 consequent aneuploidy.}

115 The cohesive function of cohesin is established in S-phase, concomitantly with DNA

116 replication. Once stabilized on the replicated genome, cohesive cohesin complexes do not turn

117 over (21). As such, loss of cohesin using our system will affect sister chromatid cohesion in all

118 cells that are in $\mathrm{S} / \mathrm{G} 2 / \mathrm{M}$ phase during the short period between TEV protease expression and

119 synthesis of RAD21-WT (Figure 1A). In addition to its canonical cohesive function, cohesin

120 has also been recently implicated in other interphase functions, including regulation of gene

121 expression (22). In contrast to the cohesive pool, these cohesin molecules are known to be

122 highly dynamic $(21,23)$. Moreover, cohesin-mediated loops were recently reported

123 "memorable" and quickly reformed upon cohesin re-establishment (24). We therefore

124 anticipated that this function should not be severely affected by our system. In sharp contrast,

125 mitotic errors induced upon cohesin cleavage are irreversible as there is no way to restore

126 cellular ploidy after a compromised round of mitosis.

128 Whereas canonical chronic mitotic perturbations lead to several rounds of mitotic failures, our 129 novel genetic system should lead to cohesion defects only in the first mitosis following the heat130 shock, as the expression of RAD21-WT should be able to rescue cohesion in the subsequent cell 131 cycle, if given enough time (Figure 1A). To confirm that our genetic system works as 132 anticipated, we focused our analysis on two different cycling tissues from the larva: the 133 developing brain and the epithelial wing discs.

134 The developing brain of Drosophila is an excellent model to study the consequences of 135 developmental aneuploidy. The well characterized cell lineages of the tissue in combination 136 with our tractable system to induce mis-segregation of chromosomes offer a unique opportunity 137 to trace the fate of aneuploid cells in real time and analyze their effect on the nervous system 138 development. Through larval development $\sim 100$ large neural stem cells called Neuroblasts 139 (Nbs) (25) located in the central brain (CB) region divide asymmetrically to self-renew and 140 generate distinct neuronal lineages via differentiating progeny (26).

141 We evaluated, by live cell imaging, mitotic fidelity in these Nbs using two independent criteria

142 to estimate the state of sister chromatid cohesion: i) the presence of single sisters (a direct

143 consequence of cohesion loss), as opposed to metaphase chromosome alignment and ii) the time

144 cells spend in mitosis, given that premature loss of sister chromatid cohesion is known to 145 activate the SAC and delay mitotic exit (Mirkovic et al., 2015).

146 As expected, the first division after the heat shock results in full cohesin cleavage in Nbs, 147 followed by cohesin rescue in subsequent divisions (Movies S1 and S2). The fast cell cycle of 148 Nbs, coupled with continued proliferation of these cells despite their abnormal genome content 149 (further discussed below), enables analysis of mitotic fidelity throughout several consecutive 
150 divisions in great detail. Consistently, in the first mitosis AHS, 95\% of Nbs contain single

151 sisters, and exhibit mitotic delay and chromosome shuffling (Figures 2A and 2B). In the

152 subsequent mitosis, however, normal cohesion is observed in $\sim 80 \%$ of the Nbs, with clear

153 metaphases and a shorter mitotic delay (Figures 2A; 2B and 2C). Finally, during the third cell

154 division AHS, the mitotic timing and the cohesive state of Nbs are comparable to heat-shocked 155 controls (Figures $2 \mathrm{~A} ; 2 \mathrm{~B}$ and $2 \mathrm{C}$ ). Similar results were obtained for larvae heat-shocked at 156 earlier stages of development (Figure S1A to $\mathrm{A}^{\prime \prime}$ ).

157 In contrast to the Nbs, in the epithelial cells of the wing disc, we observe the presence of single 158 sisters and a mitotic delay even at 48hs AHS, despite the presence of high levels RAD21-WT 159 (Figures 1A"';2D; 2E and 2F). These findings are consistent with the long cell cycle of the wing 160 discs cells $(27,28)$. The high incidence of cells affected by reversible-cohesin cleavage is also 161 consistent with a high frequency of cells in S/G2 in this tissue, estimated using the fly FUCCI 162 system (29) (Figure S3B).To fully demonstrate the ability of our tool to induce aneuploidy in an 163 acute manner in epithelial tissues we tested their regeneration capacity. In Drosophila epithelial 164 cells, multiple cellular insults, including aneuploidy, can activate the Jun N-terminal kinase 165 (JNK) signaling pathway, thus inducing the expression of pro-apoptotic genes and triggering the 166 apoptotic cascade $(9,30)$. In agreement with these studies, 24hs AHS in the wing disc, Cleaved 167 Caspase 3 (CC3) staining reveals a large population of dying cells thus reinforcing the notion 168 that cell death is mostly a consequence of the induced chromosome segregation errors and the 169 resulting aneuploidy (Figures S2A and S2A'). However, at 48hs AHS, the number of dying 170 cells decreases significantly if cohesin activity is brought back, but not if the cohesion depletion 171 by TEV is long-term (Figures S2A and S2A'). These results suggest that tissue recovery is 172 limited and only possible if the mitotic disruption is restricted in time (or cell cycle), as 173 achieved by our reversible genetic system. Although quantitative analysis was performed 174 exclusively for wing discs epithelial cells and brain Nbs, analysis of other epithelial dividing 175 tissues of the Drosophila larvae reveal a similar high incidence of single sisters 3 hs AHS, 176 implying that our system is able to induce a reversible-whole organism loss of cohesion (Figure $177 \mathrm{~S} 3 \mathrm{~A}$ ). We therefore conclude that our novel genetic tools is able to induce a single round of 178 aberrant cell division, followed by quick rescue of mitotic fidelity, across the entire organism, 179 leading to tissue-specific responses.

\section{Larvae challenged with aneuploidy during development hatch into impaired adults}

182 To understand how the entire organism would respond to such high degree of induced 183 chromosome segregation errors and consequent aneuploidy, we traced the larvae through 184 development after cohesin cleavage. For comparative analysis, we monitored eclosion rates for 185 organisms with the long-term TEV-protease cleavage system (inducing cohesin removal for $186>24 \mathrm{hs}$ ) and our newly developed system with reversible inactivation of cohesin. Both systems 
187 represent a strong insult for all the dividing tissues of the larva; therefore, we expected them to

188 be lethal in the pupa to adult transition. However, in contrast to several studies using chronic

189 mitotic perturbations $(10,31)$ flies challenged with aneuploidy, using a reversible mitotic

190 perturbation, ecloded into adult flies at high frequency, particularly if challenged up to $72 \mathrm{hs}$

191 after egg laying (AEL) (Figures 3A and Movie S3). Eclosion rates of adults were dependent on

192 the developmental stage at which cohesin was reversibly cleaved (Figure 3A). Early induction

193 of aneuploidy, at 48hs AEL, resulted in eclosion both with and without cohesin rescue.

194 However, with 72hs AEL heat-shock, there was almost no eclosion if the RAD21 protein

195 subunit was not brought back (Figure 3A). If the larvae were heat-shocked 96hs AEL, no

196 cohesin rescue resulted in dead pupae, while cohesin rescue resulted in flies trying to escape the

197 pupa, but unable to do so ("Head-out pupae") (Figures 3A and 3B).These differences in

198 developmental response to aneuploidy are likely due to increase of cell proliferation during

199 larval development $(10,32)$ (Figure S1A). Regardless of the developmental stage, all flies that

200 were able to eclode into adults after the aneuploidy challenge were completely unable to fly or

201 move normally even when showing serviceable wings and appendages (Figure $3 \mathrm{C}^{\prime}$ and Movies

202 S3 and S4).Consequently, these flies exhibited markedly shorter lifespans than their control

203 counterparts (Figure 3C). Notably, even when aneuploidy is induced at earlier stages (48h

204 AEL), thereby affecting fewer Nbs (Sup FIG1), neural tissue is not able to recover, with adults

205 displaying "lethargic" behavior (Movie S5) while having an otherwise healthy adult

206 morphology. This clearly exposes the different tissue sensitivities, showing that the developing

207 brain is extremely sensitive to any level of aneuploidy during development.

209 Few cell cycles are sufficient to induce chromosomal instability in aneuploid Neuroblasts.

210 We hypothesized that the severe motor defects in the newly hatched flies are a direct 211 consequence of aneuploidy in the developing larva brain. Recently, it has been proposed that 212 neural stem cells with unwanted karyotypes are eliminated $(10,31)$. To measure the number of 213 neural stem cells over time after aneuploidy induction, we used the Nb marker Deadpan (DPN)

214 to quantify all the nuclei with $\mathrm{Nb}$ morphology (Nb-like cells), defined based on their size, and 215 located at the central brain area (CB) per lobe. The analysis indicates that there is a gradual 216 decline in the Nbs number after the induction of aneuploidy from 12hs AHS onwards, but never 217 a complete loss of the neural stem cell population (Figures $4 \mathrm{~A}$ and $4 \mathrm{~A}^{\prime}$ ). The slow kinetics and 218 incomplete elimination of the stem cell population was quite surprising given the high levels of 219 aneuploidy generated upon cohesin loss $(\sim 100 \%)$.

220 Premature differentiation and apoptosis were suggested as the main mechanisms of aneuploid $221 \mathrm{Nb}$ elimination, reported in two recent studies $(10,31)$. However, after acute aneuploidy 222 induction in the entire $\mathrm{Nb}$ population, we found a very low frequency of cells undergoing 223 premature differentiation or cell death (Figure S4).As a proxy for premature differentiation 
events, we quantified Nb-like cells that had either lost the DPN marker or abnormally exhibit the differentiation marker Prospero (Pros) with or without co-expression of DPN (Figures S4A and $\mathrm{S} \mathrm{A}^{\prime}$, arrowheads and dashed circles).Pros is the key factor acting as a switch for the transition from stem cell self-renewal to terminal differentiation (33); therefore, this marker should not be present in Nbs. We observed that upon acute aneuploidy induction in the entire $\mathrm{Nb}$ population, there is a very low frequency of cells indicative of premature differentiation (Figure S4). These findings suggest that premature differentiation, although still taking place, is unlikely to be the major form of stem cell elimination. To estimate the levels of apoptosis, we also counted cells positive for cell death markers like CC3 and DCP1. We found a significant increase in $\mathrm{CC} 3$ positive cells in aneuploid brains (Figures S4A'; S4B' and S4B") indicating that induction of apoptosis may also contribute to the elimination of aneuploid cells, as recently proposed (10). However, CC3 and DCP1 signals rarely correspond to Nb-like cells (For CC3 staining: Control: $\mathrm{N}=4$ lobes $\mathrm{Nbs} / \mathrm{CC} 3=157 / 0(0 \%)$ and Aneuploidy induced 24hs AHS: $\mathrm{N}=9$ lobes Nbs/CC3=159/6 (3,7\%); for DCP1 staining Control: $\mathrm{N}=5$ lobes $\mathrm{Nbs} / \mathrm{CC} 3=201 / 0(0 \%)$ and Aneuploidy induced 24hs AHS: N=10 lobes Nbs/CC3=191/5 (2,6\%) (Figures S4B; S4B' and S4B", arrowheads and dashed circles), suggesting that apoptosis may not be the major cause for NB elimination. Thus, loss of stem-cell identity and/or cell death are more likely potential consequences of genome randomization, rather than a specific mechanism controlling aneuploidy in the neural stem cell population (see discussion). Supporting this idea, inhibition of apoptosis by over-expression of the baculovirus protein P35 does not rescue Nbs number per brain lobe $24 \mathrm{hs}$ after induction of aneuploidy (Figure S4C).

245 To dissect the kinetics of the aneuploid response, we took advantage of the temporal resolution of our system allowing for the tracing of aneuploid fate in real time. We restricted our analysis to $3^{\text {rd }}$ instar wandering larvae as at this stage no new Nbs are generated from the neuroepithelium (26). Induction of aneuploidy at this developmental stage, therefore, affects the entire Nbs population, which facilitates cell fate analysis. We observed a significant amount of Nbs proliferating for several days and displaying a tendency for chromosome accumulation over time (Figures 4B and 4B'). To analyze the number of chromosomes in each dividing Nbs we performed chromosome spreads and counted the number of centromeres per mitotic figure (each chromosome contains 2 centromere dots in mitosis). A single round of mitosis upon premature loss of sister chromatid cohesion should result in a maximum of 16 chromatids per Nbs, in the rare cases of complete asymmetric segregation $(0,0013 \%)$. However, chromatid numbers can reach over 32 chromatids per cell, $24 \mathrm{hs}$ and $72 \mathrm{~h}$ after loss of cohesion, at a much higher frequency (Figure 4B'). This analysis suggests that chromosome accumulation does not solely result from the initial perturbation.

259 To investigate this further, we characterized the mitotic fidelity of aneuploid Nbs. As described 260 above, mitotic divisions that immediately follow the initial perturbation do not display 
261 significant mitotic errors and the low frequency of defects observed are cohesin-related (as

262 expected from our experimental setup) (Figures 2B; 4C and 4D).

263 However, 16hs AHS, aneuploid cells start changing their behavior and a variety of mitotic

264 defects appear, becoming more frequent over time (Figures 4C and 4D'). Detailed

265 characterization of the mitotic defects arising 16hs after the induction of aneuploidy revealed

266 that majority of them $(\sim 60 \%)$ are mild, consisting of either a prolonged metaphase or a lagging

267 chromosome. However, the remaining $\sim 40 \%$ consisted of cytokinesis defects, tri-polar spindles

268 and sister chromatid cohesion defects, which are serious abnormalities that can drastically alter

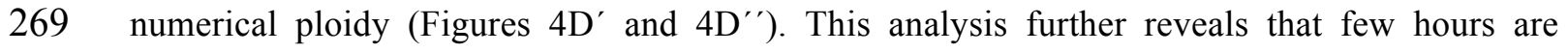

270 enough for the previously stable divisions of aneuploid karyotypes to become unstable, leading

271 to further randomization of the genome. This chromosomal instability can also contribute to

272 Nbs number decline, as catastrophic mitotic errors can result in complete loss of Nbs

273 morphology and positioning (Figure 4E). All together, we conclude that upon transient cohesin

274 loss and aneuploidy induction, neural stem cells exhibit a complex array of abnormalities as

275 consequence of their karyotype diversification, such as loss of identity, cell death, or

276 chromosomal instability, contributing to their gradual and loss over time.

277

278 Karyotype restrictions in the proliferating aneuploid Neuroblast population

279 To test if there is a selection of specific karyotypes in the population of dividing aneuploid Nbs,

280 we preformed Fluorescence In Situ Hybridization (FISH) analysis at $8 \mathrm{hs}$ and $24 \mathrm{hs}$ after

281 aneuploidy was induced. To estimate the predicted frequency of specific karyotypes we first

282 modeled the probability of each karyotype, assuming full random chromosome segregation in a

283 single round followed by a second round of random segregation in $\sim 20 \%$ of the cases (this was

284 based on our experimental observations, see Figure 2B). FISH profiles were then compared

285 with the statistical predictions (Figures 5A and 5B). The FISH profiles confirmed the propensity

286 for chromosome accumulation over time (Figures 5C and 5D). Additionally, this analysis

287 revealed that the karyotypes that can be tolerated by dividing Nbs are restricted to those

288 containing at least one of the major three chromosomes, II III or X. The rate of complete loss of

289 these chromosomes in the proliferating Nbs population was comparable to the control, and thus

290 likely a consequence of experimental error of the FISH (Figures $5 \mathrm{C}^{\prime}$ and 5D'). We concluded

291 that, although dividing aneuploid Nbs can persist in the tissue, their karyotypes have

292 restrictions, as complete loss of any of the major three chromosomes prevents their proliferation

293 in the developing brain. In contrast, other aneuploid combinations are compatible with

294 continued proliferation, particularly when Nbs gain chromosomes. 


\section{Aneuploidy elicits a stress response in the brain tissue.}

298 Our findings revealed that aneuploid cells are not promptly eliminated but instead continue to

299 proliferate within certain karyotype restrictions. This should lead not only to the maintenance of

300 aneuploid stem cells (due to $\mathrm{Nb}$ self-renewal) but also to the accumulation of differentiated

301 aneuploid progeny (note that each $\mathrm{Nb}$ divides every 2 hours (32)). We therefore tested how

302 such increase in aneuploid cells within the tissue could affect cellular physiology and influence

303 normal tissue development.

304 Several aneuploidy-associated stresses that include oxidative, metabolic, and proteotoxic stress

305 are likely to alter cellular homeostasis (3), which ultimately lead to p53 activation and a p53-

306 dependent cell-cycle arrest/senescence $(34,35)$. Interestingly, elevated levels of p53 have been

307 observed in the Central Nervous System of Down syndrome patients (36). We decided to take

308 advantage of our in vivo system to acutely induce aneuploidy to examine whether abnormal

309 karyotypes trigger a stress response in the developing Drosophila brain and if so, what is the

310 kinetics of such response. We assessed by immunohistochemistry the presence of P53 and the

311 senescence marker Dacapo (DAP, a p21/p27 homologue (37)), after the loss of cohesin and

312 consequent aneuploidy. We determined that both stress markers start to be evident at $12 \mathrm{hs}$ AHS

313 in the tissue but only at 24hs AHS significant number of cells labeled with these markers are

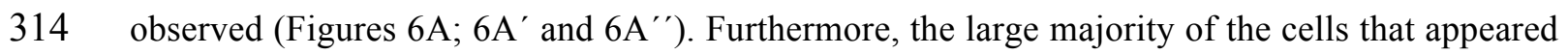

315 stress positive are not Nbs-like cells since the signal is limited to the small cells in the brain at

316 that time (Figure 6A). Nbs-like cells stained with the stress markers are noticeable only at 48hs

317 AHS (Figure 6A, arrowheads and dashed circles), suggesting that despite their aneuploid state,

318 neural stem cells are delayed at displaying an evident stress-response. We confirmed this

319 observation by quantifying the appearance of cells co-stained with the stress markers and the $\mathrm{Nb}$

320 marker DPN through time (Figure S5). We concluded that aneuploidy induction has a detectable

321 effect in the entire brain population, triggering stress responses across different cell types.

322 However, despite being promptly affected by aneuploidy induction, Nbs display a delay to this

323 cellular insult and only mount a detectable response $\sim 2$ days after becoming aneuploid.

325 Neural stemness delays aneuploidy stress response.

326 The delayed stress response (i.e. $\sim 48 \mathrm{hs}$ after induction of aneuploidy) in the neural stem cell

327 pool may imply selective stem cell tolerance for the aneuploid condition when compared to the 328 other cell types of the developing brain. To test this idea we took advantage of the brat mutant 329 condition (38). In brat mutant larvae brains, each $\mathrm{Nb}$ divides into two daughter cells grow that 330 retain Nbs properties, leading to the formation of a tumor-like neoplasm (39). We reasoned that 331 cellular stemness confers tolerance to aneuploidy, the complete occupancy of the developing 332 brain by Nbs-like cells observed in the brat mutant phenotype should be sufficient to prevent 333 the stress response observed at $24 \mathrm{hs}$ AHS. To test this idea we combined our system for acute 
334 induction of aneuploidy with brat mutations to be able to induce aneuploidy in a brat mutant

335 background and analyze the presence of stress markers at 24 and $48 \mathrm{hs}$ AHS. As predicted, DAP

336 appearance was significantly delayed in aneuploid brat mutants when compared to aneuploid

337 brains alone (Figures 6B and 6B'). The same result is observed for P53 staining (Figures S6A

338 and S6A'). Note that the Nbs marker (DPN) stains almost all the cells in brat mutant brains,

339 demonstrating the stem cell state of the entire tissue (Figures 6B and 6B').This result suggests

340 that the neural stem cell identity confers resistance to aneuploidy-associated stresses. Such

341 delayed response has the drawback that enables continuous proliferation of aneuploid neural

342 stem cells. The continued proliferation, in turn, allows for further brain growth despite presence

343 of aneuploidy. Indeed, our results show that induced aneuploidy during larval development has

344 no significant impact in the length of the brain and optic lobes in adult flies (Figure S7).

346 Protecting only the developing brain from induced aneuploidy rescues the lifespan of 347 ecloded flies.

348 Upon aneuploidy challenge, we observed a striking difference across analyzed Drosophila 349 tissues: whereas epithelial tissues like wing discs are able to regenerate from this insult (Figures 350 S2A and S2A'), a significant fraction of neural stem cells continues proliferating and becomes 351 highly chromosomally unstable (Figures 4A and 4C). These findings, together with the fact that 352 most flies that survive the developmental aneuploidy induction show severe motor defects in 353 otherwise healthy adult morphology, led us to hypothesize that the brain is the only limiting 354 tissue in response to aneuploidy during fly development.

355 To test this hypothesis, we devised a system to selectively protect only the brain from cohesin 356 removal and consequent aneuploidy. To achieve this, we complemented our reversible cohesin 357 cleavage system with brain-specific expression of RAD21-WT throughout the course of the 358 experiment (Figure 7A). In this way, TEV expression should lead to cohesion loss in all larval 359 tissues that survive solely on RAD21-TEV at the time of heat shock. In contrast, neural stem 360 cells should be resistant to this challenge, as they express both RAD21-TEV and RAD21-WT 361 (Figure 7B). Neuroblast-specific expression of RAD21-WT was achieved by the use of 362 inscutable-Gal4 (insc-Gal4) or worniu-Gal4 (wor-Gal4) drivers, to constitutively express UAS363 Rad21-wt-myc in the developing brain (Figure 7B). As expected, constitutive presence of TEV364 resistant RAD21 in the brain prevents any cohesion defects in $3^{\text {rd }}$ instar larvae Nbs (Figure 7C).

365 To confirm that the rescue of sister chromatid cohesion occurs exclusively in the brain, we 366 performed parallel characterization of the first mitotic division after the heat shock in the wing, 367 derived from the same larvae. As anticipated, full cohesin cleavage was observed in all the 368 dividing epithelial cells from the wing discs (Figure 7D). Notably, protecting only the brain 369 from developmental aneuploidy fully rescued the severe motor defects of the ecloded flies from 370 the 72hs AEL heat-shock, as demonstrated by mobility essays (Figures 7E' and Movie S6). 
371 Even more surprisingly, the brain protection was enough to rescue the lifespan of $\sim 70 \%$ of the

372 adult flies affected by organism-wide aneuploidy during development, demonstrating that the

373 brain is indeed the most sensitive tissue when challenged with aneuploidy (Figure 7E).

\section{Discussion}

377 Acute disruption of mitotic fidelity enables tracing of aneuploidy per se.

378 We developed a novel genetic tool in Drosophila to study aneuploidy in vivo. This tool enables 379 the induction of a controlled pulse of aneuploidy, at the developmental stage of choice. The 380 outcomes of using reversible perturbation are significantly different from the ones resulting 381 from chronic disruption of mitotic fidelity. Whereas chronic mitotic perturbation is 382 incompatible with organism viability, here we show a high survival rate upon controlled and 383 acute organism-wide aneuploidy challenge. The long term survival after aneuploidy challenge 384 coupled with the reversibility of the mitotic perturbation induced, overcomes one of the major 385 limitations present in other metazoan models: we were able the study the kinetics response to 386 aneuploidy across different tissues/developmental stages, focusing solely on the effects 387 aneuploidy and without the confounding variable of the mitotic perturbation used to cause 388 aneuploidy.

389 Cohesin loss and induction of aneuploidy is tolerated better by the organism if induced early in 390 development, as observed by comparing the rates of eclosion. The developing larvae are 391 progressively scaling mitotic machines, with each consecutive stage containing more divisions 392 than the previous one (32). This implies that the heat-shock at the $1^{\text {st }}$ and $3^{\text {rd }}$ instar larvae are 393 not the same, as they affect different number of dividing cells, thus generating different 394 numbers of aneuploid progeny. Although, the more parsimonious explanation for aneuploidy 395 tolerance in early development would be a quantitative one, it is also important to mention that 396 a developmental delay is observed after aneuploidy induction (e.g. delayed pupariation stage). It 397 is well known that delayed development allows the organism to adjust their growth programs 398 after disturbances $(40,41)$. This induced delay is a development-stage dependent response, as 399 some perturbations only appear to retard pupariation when induced at or before a certain stage 400 in larval development as for example, beginning of the third instar (42-44).

Chromosome mis-segregation in Neuroblasts leads to a complex array of karyotypes and 403 cellular abnormalities.

404 Neuroblasts have been used as a system to study aneuploidy response in previous studies (10, 405 31). These studies postulate two different but not mutually exclusive mechanisms of response to 406 induced aneuploidy: premature differentiation (31) and cell death by apoptosis (10). We 407 reasoned that if these are the major mechanisms of response to aneuploidy in neural stem cells, 
408 they should be detectable in high frequency after the aneuploidy induction by our acute

409 approach. Contrary to that notion, after examined in detail the kinetics of the response, both

410 premature differentiation and cell death were detected at low frequency even days after cells

411 became aneuploid. It is important to note that the degree of aneuploidy in the Nbs upon cohesin

412 loss should be around $98 \%$ due to the extensive genome shuffling prior to mitotic exit.

413 Therefore, the finding that aneuploidy does not eliminate the entire $\mathrm{Nb}$ population, strongly

414 argues against the existence of specific, active mechanisms controlling the integrity of the

415 neural stem cell genome. The more plausible explanation is that the $\mathrm{Nb}$ elimination due to

416 aneuploidy stems from a wide spectrum of abnormalities due to a randomized genome.

417 Supporting this idea, it has been shown in yeast cell-to-cell variability in cell-cycle progression

418 and robustness of multiple cellular processes even among cells harboring the same aneuploidies

$419 \quad(45)$

420 Examination of Nbs in real time after aneuploidy induction further revealed that aneuploidy is

421 sufficient to induce chromosomal instability within a short time period $(\sim 16 \mathrm{~h})$. The appearance

422 of obvious chromosomal instability, characterized by a wide range of mitotic defects, takes

423 several cell cycles after cohesin has been restored, which strongly supports the notion that

424 chromosomal instability is consequence of the abnormal karyotype and not the mitotic

425 disruption initially applied. Overall, we observe a selection towards the accumulation of

426 chromosomes, generating huge Nbs, which keep proliferating despite their increased ploidy

427 (31). Thus, our in vivo detailed examination of the aneuploid Nbs (immediately after aneuploidy

428 was induced) and kinetics of events (through several hours) clearly demonstrates that just a

429 single round of chromosome mis-segregation in these cells is enough to originate a complex

430 array of karyotypes which can lead to a variability of mitotic abnormalities.

\section{Neural stemness confers resistance to aneuploidy-associated stress response.}

433 During the last years, studies in tissue culture and yeast cells have collected solid evidence on 434 how abnormal karyotypes can remarkably alter physiology of eukaryotic cells (reviewed in (46).

435 They can lead to a different aneuploidy-associated responses that include oxidative, metabolic, 436 and proteotoxic stress which likely contribute to p53 activation and cell senescence (34). 437 However, our understanding about how aneuploidy-induced stress at the cellular level 438 influences development of tissues is very limited.

439 Our time-course assessment of classical stress response markers (P53 and DAP) following 440 chromosome mis-segregation in the brain tissue, clearly showed that aneuploidy response is not 441 immediate and takes several hours for the cells to exhibit their up-regulation (12 to 24hs AHS).

442 This delayed stress response is in agreement with recent observations in culture cells where it 443 has been shown that chromosome mis-segregation did not lead to arrest in the following G1 in 444 the vast majority of aneuploid daughter cells $(47,48)$. 
445 Interestingly, our results highlight that cell identity determines the kinetics of this stress

446 response. Aneuploidy response is specifically delayed in the neural stem cell pool (displayed

447 mainly at $\sim 48 \mathrm{hs}$ AHS) compared to the rest of the tissue, which exhibits it considerably earlier.

448 Forcing self-renewal is sufficient to delay stress response in the entire tissue, suggesting that

449 cellular stemness alone makes cells less sensitive to aneuploidy-induced stresses. Accordingly,

450 unusual resistance to altered ploidy was observed in human and mouse embryonic stem cells

451 (ESCs), mostly achieved by relaxing the cell cycle control and uncoupling the spindle

452 checkpoint from apoptosis (49). The ability of neural stem cells to continue dividing despite the

453 aneuploid karyotype dubbed them as aneuploidy "tolerant" (10). Yet, based on our findings it is

454 clear that keeping these aneuploid cells is catastrophic for normal tissue architecture and

455 development. Thus, aneuploidy may be "tolerated" better in Nbs, but the tissue as a whole is

456 unable to be functional. In contrast, the "sensitivity" of epithelial cells enables the tissue to

457 clean up and regrow properly.

459 The developing brain restricts organism recovery after induced aneuploidy.

460 Chromosomal aberrations have been long associated with neurological disorders (50). However,

461 their impact on brain development and function remains complex and poorly understood,

462 partially due to limitations of available experimental approaches. In almost all animal model

463 systems used to study aneuploidy and its consequences until now, the organisms die

464 prematurely due to the chronic disruption of mitotic fidelity to generate chromosome imbalance.

465 Therefore, it is only possible to address the short term effect of aneuploidy in nervous system

466 development, but not to understand the ultimate consequences for brain function. Our acute

467 system reversibly affects chromosome segregation to induce just a pulse of aneuploidy,

468 enabling the organism to recover from the insult and complete its development. The most

469 noticeable phenotype observed in the adult was the severe motor and behavioral defects that

470 clearly affect the lifespan of the flies, evidencing the sensitiveness of the nervous system to

471 aneuploidy. Previous studies in Drosophila have shown that the mitotic disruption in larvae Nbs

472 generates a reduction of their brain size $(10,31)$ reinforcing the idea about a link between

473 aneuploidy and microcephaly. However, our results showed that induced acute aneuploidy has

474 no significant impact in the size of the adult brain. These findings suggest that the continued

475 proliferation of neural stem cells, caused by incomplete cell elimination and delayed

476 aneuploidy-stress response, is sufficient to support the development of an apparently normal-

477 sized organ. It is conceivable that the observed normal size reflects a sample selection, as this

478 analysis was restricted to flies that survived the aneuploid challenge ( $70 \%)$. Supporting this

479 possibility, a screening performed to isolate anatomical brain mutants of Drosophila have

480 shown that mutant strains showing altered brain shape and particularly small brains are very

481 weak being mostly lethal at pupa stage (51). Despite unaltered shape and size of the adult 
482 brains, we reasoned that the neural circuits are likely impaired in those brains giving rise to the

483 adult phenotype observed in all the surviving flies.

484 In accordance with the notion of the brain as the tissue most sensitive to aneuploidy, we show

485 that preventing aneuploidy exclusively in the brain is sufficient to rescue all the behavioral

486 defects previously observed. This brain protection not only rescued motor defects but also the

487 lifespan of the flies ecloded upon 72hs AEL heat-shock, suggesting that neural tissue is the most

488 ill-equipped to deal with aneuploidy during development and impose a significant cost for the

489 organism. Several pathophysiological chromosomal disorders in humans including trisomy 21 ,

490 trisomy 18, and trisomy 13, as well as the mosaic disorder MVA (mosaic variegated

491 aneuploidy, characterized by the presence of a different number of chromosomes in some cells),

492 are well-known to display intellectual disability (50), yet the impact of the aneuploid condition

493 on brain development is still unclear $(52,53)$. Therefore, it becomes evident the necessity of

494 future studies in different animal model systems based on an acute induction of aneuploidy to

495 properly investigate its consequences for tissue development and homeostasis. These

496 approaches could help to elucidate the molecular mechanisms underlying the physiological

497 changes in stem/somatic cells generated by aneuploidy and its implications on tissue

498 development and homeostasis.

499 


\section{Fly husbandry and genetics}

504 Flies were raised using standard techniques at room temperature $\left(20-22{ }^{\circ} \mathrm{C}\right)$. We established

both chronic and the acute inactivation of cohesin complex by crossing the following genotypes: w; hspr-nlsV5TEV; Rad21(ex $\left.{ }^{3}\right) / T M 6 B$ with w;; tubpr-Rad21(550-3TEV)-EGFP, Rad21(ex $\left.{ }^{15}\right)$, polyubiq-His-RFP, and w; hspr-nlsV5TEV; Rad21(ex $\left.{ }^{3}\right)$, hspr-Gal4, UASRad21(wt)-myc/TM6B with w; tubpr-Rad21(550-3TEV)-EGFP, Rad21(ex $\left.{ }^{15}\right)$, polyubiq-His$R F P$, respectively. The progeny was then heat shocked once at $37^{\circ} \mathrm{C}$ for $45 \mathrm{~min}$ at the desired developmental stage. The correct genotype larvae were selected based on the absence of the "tubby" phenotype; the heat shocked "tubby" larvae were used as negative controls (control

512 HS). As genetic control we used the same genotypes for the induction of aneuploidy but without 513 performing the heat-shock.

514 To determine the proportion of adult eclosion, the crosses mentioned were raised in cages to monitor the time of egg collection. After 6hs collection, the plates were removed from the cages, the number of eggs counted and the plates were kept until larvae hatched. The plates were then heat-shocked at $37^{\circ} \mathrm{C}$ for $45 \mathrm{~min}$ at different larvae developmental time ( $48 \mathrm{hs} \mathrm{AEL}$, $\sim 72 \mathrm{hs}$ AEL, $\sim 96 \mathrm{hs}$ AEL and $\sim 120 \mathrm{hs}$ AEL ( $\pm 6 \mathrm{hs})$ ) and placed in a new clean plastic cage. Once they reached pupae stage ("yellow body") the pupae were gently removed with a wet brush and separated in "tubby" (control HS) and "no tubby" phenotype (condition). The different batches of pupae were placed over agar plates covered with two layers of absorbent paper to maintain the humidity and counted. The plates with the pupae were kept at room temperature until flies ecloded and the proportion of eclosion calculated.

524 To combine the induction of aneuploidy (acute cohesion inactivation) and the brat mutant genetic background we generated the following stocks: w; brat ${ }^{1} / C T G ; \operatorname{Rad} 21\left(e x^{3}\right), h s p r-G a l 4$, UAS-Rad21(wt)-myc/TM6B and w; hspr-nlsV5TEV,brat ${ }^{T S} / C T G$; tubpr-Rad21(550-3TEV)EGFP, Rad21(ex $\left.{ }^{15}\right)$, polyubiq-His-RFP. These stocks were crossed and the progeny was heat shocked once at $37^{\circ} \mathrm{C}$ for $45 \mathrm{~min}$ at the developmental stage desired and the genotype $w$; brat $^{1} /$ hspr-nlsV5TEV, brat ${ }^{T S}$; Rad21(ex $\left.{ }^{3}\right)$, hspr-Gal4, UAS-Rad21(wt)-myc/tubpr-Rad21(550$3 T E V)-E G F P, R a d 21\left(e x^{15}\right)$, polyubiq-His-RFP, was selected at larva stage based on the absent of both, GFP signal and "tubby" phenotype.

To inhibit apoptosis we induced the over-expression of the baculovirus p35 in the context of the genetic background for acute inactivation of cohesin complex. To achieve this purpose, we generated the following stock w; UAS-P35; tubpr-Rad21(550-3TEV)-EGFP 3, Rad21(ex $\left.{ }^{15}\right)$, polyubiq-His-RFP to be crossed with w; hspr-nlsV5TEV; Rad21(ex $\left.{ }^{3}\right), h s p r-G a l 4, U A S-$

$536 \operatorname{Rad} 21(w t)-m y c / T M 6 B$. The progeny was then heat-shocked once at $37^{\circ} \mathrm{C}$ for $45 \mathrm{~min}$ at the developmental stage desired. 
538 Finally, for the "brain rescue" experimental setup, we generated the following stocks: $w$; insc-

539 Gal4; tubpr-Rad21(550-3TEV)-EGFP, Rad21(ex $\left.{ }^{15}\right)$, polyubiqpr-His-RFP and w; wor-Gal4;

540 tubpr-Rad21(550-3TEV)-EGFP, Rad21(ex $\left.{ }^{15}\right)$, polyubiqpr-His-RFP. These stocks were crossed

541 with the $w$; hspr-nlsV5TEV; Rad21(ex $\left.{ }^{3}\right)$, hspr-Gal4, UAS-Rad21(wt)-myc/TM6B stock. The

542 crosses and the progeny were raised and treated as described above for the determination of the

543 eclosion proportion.

545 Table with all stocks used in this study:

\begin{tabular}{|c|c|}
\hline Stock genotype & Reference \\
\hline w; hspr-nlsV5TEV; Rad21(ex $\left.{ }^{3}\right) / T M 6 B$ & $(20)$ \\
\hline w;;tubpr-Rad21(550-3TEV)-EGFP, Rad21(ex $\left.{ }^{15}\right)$, polyubiq-His-RFP & $(54)$ \\
\hline $\begin{array}{l}\left.\text { w; hspr-nlsV5TEV; Rad21(ex }{ }^{3}\right), \text { hspr-Gal4, UAS-Rad21(wt)- } \\
\text { myc/TM6B }\end{array}$ & This study \\
\hline $\begin{array}{l}\text { w; hspr-nlsV5TEV,brat }{ }^{T S} / C T G ; \text { tubpr-Rad21(550-3TEV)-EGFP, } \\
\left.\text { Rad21(ex }{ }^{15}\right), \text { polyubiq-His-RFP }\end{array}$ & This study \\
\hline w; brat $^{1} / C T G ; \operatorname{Rad} 21\left(e^{3}\right)$, hspr-Gal4, UAS-Rad21(wt)-myc/TM6B & This study \\
\hline $\begin{array}{l}\left.\text { w; UAS-P35;tubpr-Rad21(550-3TEV)-EGFP 3, Rad21(ex }{ }^{15}\right) \text {, } \\
\text { polyubiq-His-RFP }\end{array}$ & This study \\
\hline w; HisH2AvD mRFP1 II.2/CyO; 363, CGC III.1 (R26)/TM3,Ser & $(55)$ \\
\hline brat $^{t s l} \mathrm{rdo}^{I}$ hook $^{l} \mathrm{pr}^{\mathrm{l}} / \mathrm{CyO}$ & BDSC \#3991 \\
\hline brat $^{l} \mathrm{rdo}^{l}$ hook $^{l} \mathrm{pr}^{l} / \mathrm{CyO}$ & BDSC \#3988 \\
\hline$w^{*} ; P\left[\right.$ wor.GAL4.A]2; Dr ${ }^{l} / T M 3, P[U b x-l a c Z . w+] T M 3, S b^{l}$ & BDSC \#56553 \\
\hline$w^{*} ; P[\text { GawB }]^{\text {inscMz } 1407}$ & BDSC \#8751 \\
\hline
\end{tabular}

\section{Lifespan analysis}

548 Lifespan was measured at room temperature according to standard protocols. In brief, newly ecloded animals ( 0 to 3 days) were collected (50 per genotype: "control", "Aneuploidy" and "Aneuploidy + brain rescue"), and then placed in vials (up to 10 per vial), and transferred to fresh vials every two days. Survival was recorded for each vial. Due to the reduced mobility of the aneuploidy genotypes, we scored flies stacked in the food as death events in all the vials

553 analyzed. We created survival curves with Prism 5.00 for Windows (GraphPad Software, San

554 Diego, CA, USA) using the method of Kaplan and Meier.aq 
556 For climbing assay flies were anesthetized with $\mathrm{CO} 2$, separated in groups of around twenty

557 adults ( 3 replicas for each genotype) and allowed to recover for $2 \mathrm{hs}$ before beingsubjected to a

558 climbing assay. Briefly, the groups of over twenty flies were placed in an empty climbing vial

559 and then tapped down to the bottom. They were allowed to climb past the halfway point from

560 the bottom of the vial for 30 seconds $(10 \mathrm{~cm})$. The number of flies above the $10 \mathrm{~cm}$ mark was recorded as a percentage of flies able to climb.

\section{Histology}

564 Briefly, flies were anesthetized with $\mathrm{CO} 2$ and then were placed gently in agarose blocks to

565 immobilize them and prevent any damage to the head or eyes. The agarose blocks with the flies

566 were immersed in Carnoy fixative overnight, at $4^{\circ} \mathrm{C}$. The next day the Carnoy solution was

567 removed and three $70 \%$ ethanol washes were performed. Immediately after, the flies were

568 decapitated and the heads were oriented one by one in melted $2 \%$ agarose to guarantee similar

569 orientation of the tissue sections. Agarose blocks were then processed, embedded, the whole

570 head was sectioned into 5um-thick sequential sections and stained with Hematoxylin \& Eosin.

571 The histology was performed in the Histopathology unit at Instituto Gulbenkian de Ciência and

572 the slides were analyzed by a pathologist with a DMLB2 microscope (Leica). Images were

573 acquired with a DFC320 camera (Leica) and NanoZoomer-SQ Digital slide scanner

574 (Hamamatsu).

575

576 Live-cell imaging

577 Larvae $3^{\text {rd }}$ instar brains were dissected in Schneider medium supplemented with $10 \%$ FBS and 578 intact brains were mounted on a glass-bottom dish (MakTek), covered with an oxygen579 permeable membrane (YSI membrane kit), and sealed with Voltalef oil 10S (VWR). This 580 procedure allowed long-term imaging of brains for periods up to 10 hours.

581 For imaging of imaginal discs and early instar larvae brains, tissues were dissected in Schneider

582 medium with $10 \%$ FBS. Dissected discs were placed and oriented in a $200 \mu 1$ drop of medium at 583 the bottom of a glass-bottom dish (MakTek).

584 Live imaging was performed on a spinning disc confocal using imaged on a Revolution XD 585 microscope (Andor, UK) equipped with immersion a $60 \mathrm{x}$ glycerol-immersion $1.30 \mathrm{NA}$ 586 objective (Leica Microsystems) and a iXon Ultra 888 1024*1024 EMCCD (Andor, UK). 25-35 587 Z-series optical sections were acquired $0.5-1 \mu \mathrm{m}$ apart.

\section{Brain spreads and Immunofluorescence}

590 For brain spreads and immunofluorescence, $3^{\text {rd }}$ instar larvae brains were dissected in PBS, 591 incubated with $100 \mu \mathrm{M}$ colchicine for one hour, hypotonic shocked in $0.5 \%$ sodium citrate for 

PBS) placed on top of a siliconized coverslip. After 30 seconds, the brains were squashed between the coverslip and a slide, allowed to fix for an additional $1 \mathrm{~min}$ and then placed in liquid nitrogen. Slides were further extracted with $0.1 \%$ Triton-X100 in PBS for $10 \mathrm{~min}$, and used for immunofluorescence following standard protocols. Primary antibodies were rat antiCID (gift from Claudio E. Sunkel) used at 1:2000, Cleaved Drosophila Dcp-1 (Asp216)

598 Antibody (1:300) \# 1679578S (Cell Signaling Technology), Cleaved Caspase-3 (Asp175)

599 Antibody \#9661 (1:300) (Cell Signaling Technology), Anti-Deadpan antibody \#ab195173

600 (1:1500) (Abcam). Secondary antibodies conjugated with fluorescent dyes from Alexa series

601 (Invitrogen) were used according to the manufacturer's instructions.

602 Third instar wing imaginal disc fixation and staining, as well as immunofluorescence of whole

603 brains was performed using standard procedures (Lee and Treisman, 2001). Briefly, third instar

604 larvae wing disc tissue (still attached to the larva body) was fixed on ice for $30 \mathrm{~min}$. The

605 fixative consisted of 4\% formaldehyde (Polysciences) in 1X PEM buffer solution. Following 606 were washed by gentle agitation three times for $20 \mathrm{~min}$ in PBS-T (1x PBS $+0.1 \%$ Triton X607 100). Primary antibodies incubation was performed overnight at $4{ }^{\circ} \mathrm{C}$ in PBS-T supplemented 608 with $1 \%$ BSA and $1 \%$ donkey serum. The following day, the tissues were washed again and 609 incubated for $2 \mathrm{~h}$ at room temperature with the appropriate secondary antibodies diluted in PBS$610 \mathrm{~T}$ solution. Finally, after the wash of secondary antibodies, wing discs were mounted in 611 Vectashield (Vector Laboratories). Fluorescence images were acquired with a $\times 40$ HCX PL 612 APO CS oil immersion objective (numerical aperture: 1.25-0.75) on a Leica SP5 confocal 613 microscope.

614

\section{Fluorescence In Situ Hybridization}

616 Brains from $3^{\text {rd }}$ instar larvae were dissected in PBS, incubated with $100 \mu \mathrm{M}$ colchicine for one 617 hour, and transferred to $0.5 \%$ sodium citrate solution for 3-4 minutes. Then, the brains were 618 transferred to a fixative containing 11:11:2 Methanol: Acetic Acid: MQ Water, for 30 seconds 619 before being placed in a droplet of $45 \%$ Acetic acid for 2 minutes, squashed and transferred to 620 liquid Nitrogen. Then, the coverslip was removed and the slide incubated in absolute ethanol for $62110 \mathrm{~min}$ at $-20^{\circ} \mathrm{C}$ (Freezer incubation). The slides were air dried at $4{ }^{\circ} \mathrm{C}$. (20 minutes). The slides 622 were dehydrated at room temperature in 70\%,90\% and absolute ethanol for 3 minutes, prior to 623 DNA denaturation in $70 \%$ formamide- $2 x S C C$ solution for 2 minutes at $70{ }^{\circ} \mathrm{C}$. This is done on 624 the thermomixer set at $70{ }^{\circ} \mathrm{C}$ with a formamide solution heated to $70^{\circ}$. Then, the slides were 625 transferred to cold $70 \%$ Ethanol $\left(-20^{\circ} \mathrm{C}\right)$ and dehydrated at room temperature in $90 \%$ and 626 absolute ethanol for $3 \mathrm{~min}$. FISH probes were denatured in the hybridization buffer at $92 \mathrm{C}$ for 3 $627 \mathrm{~min}$.Hybridization was done over-night at $37^{\circ} \mathrm{C}$ using $30 \mathrm{ul}$ of FISH hybridization buffer/probe 628 mix per slide. Hybridization buffer: $20 \%$ dextran sulfate in $2 \mathrm{x}$ SCCT/50\% 
629 Formamide $/ 0,5 \mathrm{mg} / \mathrm{ml}$ Salmon sperm DNA. Then, slides were washed $3 \times 5 \mathrm{~min}$ in $50 \%$

630 formamide- $2 \mathrm{xSCC}$ at $42{ }^{\circ} \mathrm{C}$ and $3 \times 5 \mathrm{~min}$ in $0.1 \times \mathrm{SCC}$ at $60^{\circ} \mathrm{C}$. These steps are done on the

631 thermomixer, with the solutions previously heated to desired temperatures. Finally, the slides

632 are washed in PBS, and mounted in Vecta shield with DAPI. The probes were used in the final

633 concentration of $70 \mathrm{Nm}$ in hybridization buffer. Probes used were: Chr_X (359 bp satellite

634 DNA) A546-GGGATCGTTAGCACTGGTAATTAGCTGC, and Ch_3 (dodeca satellite DNA)

635 Cy5-ACGGGACCAGTACGG DNA probes, Chr_2 A488-(AACAC).

636

637 Western-blot

638 To analyze RAD21 protein amounts, Drosophila tissues were dissected in PBS and

639 homogenized with a pestle in Sample buffer. Samples were centrifuged, and boiled for 5

640 minutes in 2x Sample Buffer. Samples were loaded on a $13 \%$ SDS-gel for electrophoresis and

641 and then transferred to nitrocellulose membranes. Western-blot analysis was performed

642 according to standard protocols using the following antibodies: anti- $\alpha$-tubulin $(1: 50.000$,

643 DM1A, Sigma-Aldrich Cat\# T9026), guinea pig anti-Rad21 (Heidmann et al., 2004) and

644 V5 Tag Mouse Monoclonal Antibody (Novex $\left.{ }^{\circledR}\right)$.

645

646 Image analysis

647 Imaging analysis was performed using FIJI software (Schindelin et al., 2012). For z-

648 projections slices were stacked into maximum intensity (10 frames, $2 \mu \mathrm{m}$ each). Some pictures

649 were rotated and/or flipped to orient them in the same way.

650

651 Statistical analysis

652 Statistical analysis and graphic representations were performed using Prism 5.00 for Windows

653 (GraphPad Software, San Diego, CA, USA). Unpaired t test or one-way ANOVA (using the

654 Bonferroni's multiple comparison) were applied depending the measurements analyzed in the corresponding experiment. Sample size details are included in the respective plotted graphs.

\section{Acknowledgments}

658 We thank S. Heidmann and the Bloomington Stock Center for fly strains, the Advance Imaging 659 Unit and Fly Facility, Histopathology of Instituto Gulbenkian de Ciencia for technical 660 assistance, and C. Homem, F. Janody and M. Bettencourt-Dias, and all the members of the 661 RAO laboratory for discussions and comments. MM was supported by a Fundação para a 662 Ciência e Tecnologia, FCT, fellowship (SFRH /BD/52438/2013). This work was supported by 663 the following grants awarded to RAO: FCT Investigator grant 664 (IF/00851/2012/CP0185/CT0004), EMBO Installation Grant (IG2778) and European Research 665 Council Starting Grant (ERC-2014-STG-638917). 
1. Ambartsumyan G, Clark AT. Aneuploidy and early human embryo development. Hum Mol Genet. 2008;17(R1):R10-5.

2. Holland AJ, Cleveland DW. Boveri revisited: chromosomal instability, aneuploidy and tumorigenesis. Nat Rev Mol Cell Biol. 2009;10(7):478-87.

672 3. Santaguida S, Amon A. Short- and long-term effects of chromosome missegregation and aneuploidy. Nat Rev Mol Cell Biol. 2015;16(8):473-85.

674 4. Sheltzer JM, Ko JH, Replogle JM, Habibe Burgos NC, Chung ES, Meehl CM, et al. Single-chromosome Gains Commonly Function as Tumor Suppressors. Cancer Cell. 2017;31(2):240-55.

5. Gordon DJ, Resio B, Pellman D. Causes and consequences of aneuploidy in cancer. Nat Rev Genet. 2012;13(3):189-203.

6. Lara-Gonzalez P, Westhorpe FG, Taylor SS. The spindle assembly checkpoint.

681 7. Knouse KA, Wu J, Whittaker CA, Amon A. Single cell sequencing reveals low levels of aneuploidy across mammalian tissues. Proc Natl Acad Sci U S A. 2014;111(37):13409-14.

\section{Pfau SJ, Silberman RE, Knouse KA, Amon A. Aneuploidy impairs} hematopoietic stem cell fitness and is selected against in regenerating tissues in vivo. Genes Dev. 2016;30(12):1395-408.

9. Dekanty A, Barrio L, Muzzopappa M, Auer H, Milan M. Aneuploidy-induced delaminating cells drive tumorigenesis in Drosophila epithelia. Proc Natl Acad Sci U S A. 2012;109(50):20549-54.

10. Poulton JS, Cuningham JC, Peifer M. Centrosome and spindle assembly checkpoint loss leads to neural apoptosis and reduced brain size. J Cell Biol. 2017;216(5):1255-65.

11. Ly P, Cleveland DW. Interrogating cell division errors using random and chromosome-specific missegregation approaches. Cell Cycle. 2017:1-7.

12. Hassold T, Hunt P. To err (meiotically) is human: the genesis of human aneuploidy. Nat Rev Genet. 2001;2(4):280-91.

13. Guacci V, Yamamoto A, Strunnikov A, Kingsbury J, Hogan E, Meluh P, et al. Structure and function of chromosomes in mitosis of budding yeast. Cold Spring Harb Symp Quant Biol. 1993;58:677-85.

700 14. Michaelis C, Ciosk R, Nasmyth K. Cohesins: chromosomal proteins that prevent premature separation of sister chromatids. Cell. 1997;91(1):35-45.

15. Mirkovic M, Oliveira RA. Centromeric Cohesin: Molecular Glue and Much More. Prog Mol Subcell Biol. 2017;56:485-513.

704 16. Nasmyth K, Haering CH. Cohesin: its roles and mechanisms. Annu Rev Genet. 2009;43:525-58.

706 17. Haering CH, Farcas AM, Arumugam P, Metson J, Nasmyth K. The cohesin ring concatenates sister DNA molecules. Nature. 2008;454(7202):297-301.

708 18. Ivanov D, Nasmyth K. A topological interaction between cohesin rings and a circular minichromosome. Cell. 2005;122(6):849-60. 19. Mirkovic M, Hutter LH, Novak B, Oliveira RA. Premature Sister Chromatid

712 System-Level Feedback. Cell Rep. 2015;13(3):470-8.

713 20. Pauli A, Althoff F, Oliveira RA, Heidmann S, Schuldiner O, Lehner CF, et al.

714 Cell-type-specific TEV protease cleavage reveals cohesin functions in Drosophila 715 neurons. Dev Cell. 2008;14(2):239-51. 
716 21. Gerlich D, Koch B, Dupeux F, Peters JM, Ellenberg J. Live-cell imaging reveals

717 a stable cohesin-chromatin interaction after but not before DNA replication. Curr Biol.

718 2006;16(15):1571-8.

719 22. van Ruiten MS, Rowland BD. SMC Complexes: Universal DNA Looping

720 Machines with Distinct Regulators. Trends Genet. 2018;34(6):477-87.

721 23. Eichinger CS, Kurze A, Oliveira RA, Nasmyth K. Disengaging the Smc3/kleisin

722 interface releases cohesin from Drosophila chromosomes during interphase and mitosis.

723 EMBO J. 2013;32(5):656-65.

724 24. Rao SSP, Huang SC, Glenn St Hilaire B, Engreitz JM, Perez EM, Kieffer-Kwon

725 KR, et al. Cohesin Loss Eliminates All Loop Domains. Cell. 2017;171(2):305-20 e24.

726 25. Urbach R, Technau GM, Breidbach O. Spatial and temporal pattern of

727 neuroblasts, proliferation, and Engrailed expression during early brain development in

728 Tenebrio molitor L. (Coleoptera). Arthropod Struct Dev. 2003;32(1):125-40.

729 26. Homem CC, Knoblich JA. Drosophila neuroblasts: a model for stem cell

730 biology. Development. 2012;139(23):4297-310.

731 27. Milan M, Campuzano S, Garcia-Bellido A. Cell cycling and patterned cell

732 proliferation in the wing primordium of Drosophila. Proc Natl Acad Sci U S A.

733 1996;93(2):640-5.

734 28. Neufeld TP, de la Cruz AF, Johnston LA, Edgar BA. Coordination of growth and cell division in the Drosophila wing. Cell. 1998;93(7):1183-93.

29. Zielke N, Korzelius J, van Straaten M, Bender K, Schuhknecht GF, Dutta D, et al. Fly-FUCCI: A versatile tool for studying cell proliferation in complex tissues. Cell 738 Rep. 2014;7(2):588-98.

739 30. Milan M, Clemente-Ruiz M, Dekanty A, Muzzopappa M. Aneuploidy and 740 tumorigenesis in Drosophila. Semin Cell Dev Biol. 2014;28:110-5.

741 31. Gogendeau D, Siudeja K, Gambarotto D, Pennetier C, Bardin AJ, Basto R. 742 Aneuploidy causes premature differentiation of neural and intestinal stem cells. Nat 743 Commun. 2015;6:8894.

744 32. Ito K, Hotta Y. Proliferation pattern of postembryonic neuroblasts in the brain of Drosophila melanogaster. Dev Biol. 1992;149(1):134-48.

33. Choksi SP, Southall TD, Bossing T, Edoff K, de Wit E, Fischer BE, et al. Prospero acts as a binary switch between self-renewal and differentiation in Drosophila neural stem cells. Dev Cell. 2006;11(6):775-89.

749 34. Kruiswijk F, Labuschagne CF, Vousden KH. p53 in survival, death and

750 metabolic health: a lifeguard with a licence to kill. Nat Rev Mol Cell Biol.

$751 \quad 2015 ; 16(7): 393-405$.

752 35. Thompson SL, Compton DA. Proliferation of aneuploid human cells is limited by a p53-dependent mechanism. J Cell Biol. 2010;188(3):369-81.

36. Liao JM, Zhou X, Zhang Y, Lu H. MiR-1246: a new link of the p53 family with cancer and Down syndrome. Cell Cycle. 2012;11(14):2624-30.

37. Lane ME, Sauer K, Wallace K, Jan YN, Lehner CF, Vaessin H. Dacapo, a cyclin-dependent kinase inhibitor, stops cell proliferation during Drosophila development. Cell. 1996;87(7):1225-35.

759 38. Arama E, Dickman D, Kimchie Z, Shearn A, Lev Z. Mutations in the beta-

760 propeller domain of the Drosophila brain tumor (brat) protein induce neoplasm in the

761 larval brain. Oncogene. 2000;19(33):3706-16.

762 39. Betschinger J, Mechtler K, Knoblich JA. Asymmetric segregation of the tumor

763 suppressor brat regulates self-renewal in Drosophila neural stem cells. Cell.

764 2006;124(6):1241-53. 
765 40. Gontijo AM, Garelli A. The biology and evolution of the Dilp8-Lgr3 pathway:

766 A relaxin-like pathway coupling tissue growth and developmental timing control. Mech

767 Dev. 2018.

768 41. Hackney JF, Cherbas P. Injury response checkpoint and developmental timing in

769 insects. Fly (Austin). 2014;8(4):226-31.

770 42. Garelli A, Gontijo AM, Miguela V, Caparros E, Dominguez M. Imaginal discs secrete insulin-like peptide 8 to mediate plasticity of growth and maturation. Science. 2012;336(6081):579-82.

43. Halme A, Cheng M, Hariharan IK. Retinoids regulate a developmental checkpoint for tissue regeneration in Drosophila. Curr Biol. 2010;20(5):458-63.

44. Simpson P, Berreur P, Berreur-Bonnenfant J. The initiation of pupariation in Drosophila: dependence on growth of the imaginal discs. J Embryol Exp Morphol. 1980;57:155-65.

778 45. Beach RR, Ricci-Tam C, Brennan CM, Moomau CA, Hsu PH, Hua B, et al. Aneuploidy Causes Non-genetic Individuality. Cell. 2017;169(2):229-42 e21.

780 46. Santaguida S, Vasile E, White E, Amon A. Aneuploidy-induced cellular stresses limit autophagic degradation. Genes Dev. 2015;29(19):2010-21. Chromosome Mis-segregation Generates Cell-Cycle-Arrested Cells with Complex Karyotypes that Are Eliminated by the Immune System. Dev Cell. 2017;41(6):638-51 e5.

48. Soto M, Raaijmakers JA, Bakker B, Spierings DCJ, Lansdorp PM, Foijer F, et al. p53 Prohibits Propagation of Chromosome Segregation Errors that Produce Structural Aneuploidies. Cell Rep. 2017;19(12):2423-31.

789 49. Mantel C, Guo Y, Lee MR, Kim MK, Han MK, Shibayama H, et al.

790 Checkpoint-apoptosis uncoupling in human and mouse embryonic stem cells: a source of karyotpic instability. Blood. 2007;109(10):4518-27.

792 50. Bushman DM, Chun J. The genomically mosaic brain: aneuploidy and more in neural diversity and disease. Semin Cell Dev Biol. 2013;24(4):357-69.

794 51. Heisenberg B. Isolation of Anatomical Brain Mutants of Drosophila

795 by Histological Means. Z Naturforsch 34 c, 143 - 147 (1979) 1979.

796 52. Oromendia AB, Amon A. Aneuploidy: implications for protein homeostasis and 797 disease. Dis Model Mech. 2014;7(1):15-20.

798 53. Ricke RM, van Deursen JM. Aneuploidy in health, disease, and aging. J Cell 799 Biol. 2013;201(1):11-21.

800 54. Oliveira RA, Kotadia S, Tavares A, Mirkovic M, Bowlin K, Eichinger CS, et al.

801 Centromere-independent accumulation of cohesin at ectopic heterochromatin sites

802 induces chromosome stretching during anaphase. PLoS Biol. 2014;12(10):e1001962.

803 55. Oliveira RA, Hamilton RS, Pauli A, Davis I, Nasmyth K. Cohesin cleavage and 804 Cdk inhibition trigger formation of daughter nuclei. Nat Cell Biol. 2010;12(2):185-92. 


\section{Figure Legends}

\section{8 \#Main Figures:}

\section{$809 \quad$ Figure 1}

810 Reversible system for acute cohesin depletion and generation of aneuploidy in the 811 developing Drosophila.

812 A to A": A- The system relies on the cleavage of RAD21 Cohesin subunit (RAD21-TEV) by 813 the heat-shock induced expression of TEV protease. Cohesin cleavage is rapid and the RAD21814 TEV is completely depleted within hours after the heat-shock (see western blots $\mathrm{A}^{\prime}, \mathrm{A}^{\prime \prime}$, 815 RAD21 upper band). Cells entering into mitosis with loss of cohesion experience premature 816 sister chromatid separation, chromosome shuffling and as consequence generation of 817 aneuploidy daughter cells with a theoretical rate of nearly $100 \%$. To restrict mitotic failure to a 818 single cell cycle, shortly after aneuploidy has been generated, cohesion is rescued by inducing 819 the expression of TEV-resistant RAD21 protein (RAD21-WT). The RAD21-WT subunit is 820 reliant on heat-shock Gal4 protein expression, coupled to the expression of the UAS-RAD21821 WT and occurs within hours after the heat-shock (see western blots A', A", RAD21 lower 822 band). A'and $\mathbf{A}^{\prime \prime}$ - Western blots displaying RAD21-TEV cleavage and RAD21-WT restoring 823 cohesin function in the $3^{\text {rd }}$ instar larvae brains and wing discs. Tissues samples were taken at 824 different hours after the heat-shock induction (AHS). V5 antibody evidences the TEV protease 825 expression (from hspr-nlsV5TEV) and Tubulin antibody was used as loading control.

826

\section{$827 \quad$ Figure 2}

828 Kinetics of reversible loss of cohesin in the $3^{\text {rd }}$ instar brains and the wing discs.

829 A- Stills from live imaging of Nbs after the heat-shock (full movies available in supplementary

830 movie 1 and 2). Transient loss of cohesion results in a round of defective mitosis and genome 831 shuffling $\left(1^{\text {st }}\right.$ division AHS $)$. After this round of division, the following mitosis $\left(2^{\text {nd }}\right.$ and $3^{\text {rd }}$ 832 divisions AHS) shows the restoring of cohesion function and mitotic fidelity in larvae Nbs. 833 Dashed circles display the mitotic figures of the dividing Nbs.

834 B- Quantification of Cohesive states of $3^{\text {rd }}$ instar larvae Nbs following RAD21-TEV cleavage 835 and RAD21-WT restoring of cohesin function. More than $80 \%$ of the $2^{\text {nd }}$ divisions AHS are 836 already totally or partially cohesed.

837 C- Quantification of mitotic timing and delay caused by Spindle Assembly Checkpoint 838 activation after RAD21-TEV cleavage and RAD21-WT rescue in the $3^{\text {rd }}$ instar Nbs (2 to 24hs 839 AHS). $2^{\text {nd }}$ divisions AHS evidence a significant reduction in the mitotic timing as a 840 consequence of the rescue of cohesin function. $* * * *=\mathrm{P}<0.0001$. 
841 D- Cohesin cleavage in the Wing disc of $3^{\text {rd }}$ instar larvae. Stills from live imaging showing

842 single chromatids during mitosis. Dashed circles display epithelial cells from the wing disc

843 pouch undergoing mitosis with loss of cohesion.

844 E- Quantification of Cohesive states of divisions in the $3^{\text {rd }}$ instar larvae wing disc following

845 RAD21-TEV cleavage and Rad21-WT rescue from $2 \mathrm{~h}$ to $80 \mathrm{~h}$ after heat-shock. Given the long 846 cell cycle and the heterogeneous rate of division of cells in this tissue the presence of single 847 sisters can still be observed up to $72 \mathrm{~h}$ after the heat-shock.

848 F- Quantification of mitotic timing and delay caused by Spindle Assembly Checkpoint 849 activation after Rad21-TEV cleavage and Rad21-WT rescue in the $3^{\text {rd }}$ instar wing discs from 2 850 to $80 \mathrm{hs}$ AHS.

851 In all panels $\mathrm{n}=$ number of cells. $* * * *=\mathrm{P}<0.0001 ; * *=\mathrm{P}<0.01$.

852

$853 \quad$ Figure 3

854 Larvae challenged with organism-wide aneuploidy hatch into adult flies with severe motor defects and reduced lifespan.

856 A- Percentages of adult eclosions according to the stage of development at which reversible loss 857 of cohesin (aneuploidy) was inducted (48, 72, 96 and 120hs after egg laying, AEL). n= number 858 of flies.

859 B- Picture showing "head out pupae" flies as a result of 96hs AEL induced aneuploidy

860 C to $\mathbf{C}^{\prime}$ : $\mathbf{C}$ - Kaplan-Meier survival curves showing fractional survival as a function of time.

861 Ecloded flies with 72hs AEL induced aneuploidy showed reduced lifespan when compared with 862 control flies (only heat-shocked). $\mathbf{C}^{\prime}$ - Climbing assay comparing adult flies with $72 \mathrm{hs}$ induced 863 aneuploidy and control flies. Percentage of climb success was plotted over the halfway point 864 (10 cm). Ecloded flies with 72hs AEL induced aneuploidy showed impaired motor behavior.

\section{$866 \quad$ Figure 4}

867 Aneuploidy results Neuroblast loss, chromosomal instability and accumulation of 868 chromosomes.

869 A to $\mathbf{A}^{\prime}:$ A- Nbs counts at the central brain (dashed shape) in $3^{\text {rd }}$ instar lobe brains assessed by 870 immunofluorescence with the Nbs marker Deadpan (DPN). $\mathbf{A}^{\prime}$ - Nbs were quantified based on 871 the correlation between morphology and positive signal for DPN at 12, 24 and 48hs AHS. 872 Reversible loss of cohesion and aneuploidy are followed by a reduction in Nbs numbers, but not 873 a complete loss of the neural stem cell pool. $\mathrm{n}=$ number of lobe brains. $* * *=\mathrm{P}<0.001$; **** $=$ $874 \mathrm{P}<0.0001 ; \mathrm{ns}=$ no significant. Scale $\mathrm{bar}=40 \mu \mathrm{m}$.

875 B to B': B- Chromosome counts were assessed by CID immunofluorescence (Centromere 876 counts) in $3^{\text {rd }}$ instar Nbs arrested at metaphase with Colchicine (dashed circle) at 24 and $72 \mathrm{hs}$ 
877 AHS. B'- Aneuploid Nbs accumulate chromosomes through time. $\mathrm{n}=$ number of cells. $* * * *=$

$878 \mathrm{P}<0.0001 ; * *=\mathrm{P}<0.01$. Scale bar $=5 \mu \mathrm{m}$.

879 C- Assessment of mitotic defects after aneuploidy induction from 2 to $48 \mathrm{hs}$ AHS. Chromosome

880 instability arises shortly after aneuploidy induction (red dashed box). $\mathrm{n}=$ number of cells.

881 D to $\mathbf{D}^{\prime \prime}$ : D and $\mathbf{D}^{\prime}$ - Profile of mitotic errors as a consequence of reversible cohesin depletion 882 and consequent aneuploidy from 2 to $12 \mathrm{hs}$ AHS and 16 to $48 \mathrm{hs}$ AHS. D"- Stills from live 883 imaging documenting mitotic abnormalities in aneuploid Nbs (dashed circles). $n=$ number of 884 cells.

885 E- Graph displaying the rate of catastrophic mitotic events which result in Nbs loss from 2 to

886 48hs AHS. Chromosomal instability can result in complete loss of Nbs morphology. $n=$ number 887 of cells.

\section{$889 \quad$ Figure 5}

890 Karyotype restrictions in the proliferating aneuploid Nbs population

891 A- Panels of Fluorescent in situ Hybridization (FISH) of aneuploid Nbs and Control. Schematic 892 of the FISH probe chromosome labeling.

893 B- Theoretical segregation of sister chromatids of a given chromosome after cohesion loss, 894 assuming segregation to be random (95\% in the first mitosis, $20 \%$ in the second mitosis). We 895 assumed that each chromatid segregates independently of all other chromatids and with equal 896 probability to the mother and daughter cell. Given this, and for one cell division (mitosis) with 897 diploid cells (4 chromatids for each chromosome) the distribution of the number of copies of 898 each chromatid per cell is given by a Binomial distribution with 4 trials and probability of 899 success of 0,5 .

900 C to $\mathbf{C}^{\prime}$ : $\mathbf{C}$ - Frequency distribution of chromosome copy per Nbs, 8hs after aneuploidy 901 induction. $\mathbf{C}^{\prime}$ - Calculated theoretical loss rate for chromosome II, III or X, and the observed 902 frequency of loss of any of these three chromosomes in the proliferating aneuploid $\mathrm{Nbs}(\mathrm{n}>250$ 903 over eight brains analyzed per condition).

904 D to D': D- Frequency distribution of chromosome copy per Nbs, 24hs after aneuploidy 905 induction. D'- Calculated theoretical loss rate for chromosome II, III or X, and the observed 906 frequency of loss of any of these three chromosomes in the proliferating aneuploid $\mathrm{Nbs}(\mathrm{n}>250$ 907 over eight brains analyzed per condition).

\section{$909 \quad$ Figure 6}

910 Aneuploidy-induced stress response is delayed in the neural tissue.

911 A to $\mathbf{A}^{\prime \prime}$ : A- Kinetics of the aneuploidy induced stress response at 6, 12, 24, and 48hs AHS; 912 assessed by immunofluorescence of canonical markers P53 and Dacapo (DAP). Nbs show a 913 delayed aneuploidy stress response at 48hs AHS (arrowheads with dashed circles). $\mathbf{A}^{\prime}$ and $\mathbf{A}^{\prime \prime}-$ 
914 Counts of overall numbers of p53 and DAP signals per lobe, displaying a significant increase

915 from 24hs AHS. ; *** $=\mathrm{P}<0.001 ; * * * *=\mathrm{P}<0.0001$. Scale bar $=40 \mu \mathrm{m}$. z-proj (z projection).

916 B to $\mathbf{B}^{\prime}$ : B- Pictures from fixed samples of $3^{\text {rd }}$ instar larvae lobe brains showing the

917 immunofluorescence of the stress marker DAP and the Nbs marker (DPN) at 24 and 48hs after

918 induction of aneuploidy. B'- Quantification of relative DAP positive signal per lobe area from

91924 to $48 \mathrm{hs}$ AHS tissues. Brat mutant lobe brains showed a clear reduction in the presence of the

920 aneuploidy induced stress marker DAP at 24hs AHS. $\mathrm{n}=$ number of lobe brains. $* * * *=$

$921 \mathrm{P}<0.0001$. Scale bar $=40 \mu \mathrm{m}$. z-proj (z projection).

922

\section{$923 \quad$ Figure 7}

924 Protecting only the developing brain from induced aneuploidy rescues the adult lifespan.

925 A- Graphic scheme depicting how the developing brain is protected from the loss of cohesion

926 and induction of aneuploidy upon the constitutive expression of the RAD21-WT driven by Nbs

927 specific Gal4 (Insc-Gal4 or Wor-Gal4). In contrast, the rest of the dividing tissues from the

928 larva experience the acute inactivation of cohesin complex (by TEV cleavage of RAD21-TEV)

929 after the heat-shock.

930 B- Western blots showing the expression of both the cleavable (RAD21-TEV) and non-

931 cleavable RAD21 (RAD21-WT) in $3^{\text {rd }}$ instar brains. Insc-Gal4 and Wor-Gal4 drivers result in

932 expression of RAD21-WT in the $3^{\text {rd }}$ instar brain before the heat-shock.

933 C- Quantification of Cohesive states of $3^{\text {rd }}$ instar larvae Nbs and epithelial cells from wing discs

934 following heat-shock. Insc-Gal4 protects the brain from cohesin loss, but has no effect in the 935 wing disc.

936 D- Live imaging cohesion profiles of $\mathrm{Nbs}$ and epithelial cells during mitotic divisions after the

937 heat-shock. Insc-Gal4 prevents mitotic delay caused by cohesion loss in the Nbs.

938 E to $\mathbf{E}^{\prime}$ : E- Kaplan-Meier survival curves showing fractional survival as a function of time.

939 Protection of the brain tissue from the induced aneuploidy rescues the adult lifespan of the

940 ecloded flies with $72 \mathrm{hs}$ AEL heat-shock. E'- Climbing assay of adult flies. Percentage of climb

941 success was plotted over the halfway point $(10 \mathrm{~cm})$. Protection of the brain tissue from induced

942 aneuploidy rescues motor defects of the ecloded flies with the 72hs AEL heat-shock. 
DoRxiv preprint doi: https://doi.org/10.1101/392746; this version posted August 15, 2018. The copyright holder for this preprint (which was not certified by peer review) is the author/funder, who has granted bioRxiv a license to display the preprint in perpetuity. It is made available under ACC-BY 4.0 International license.

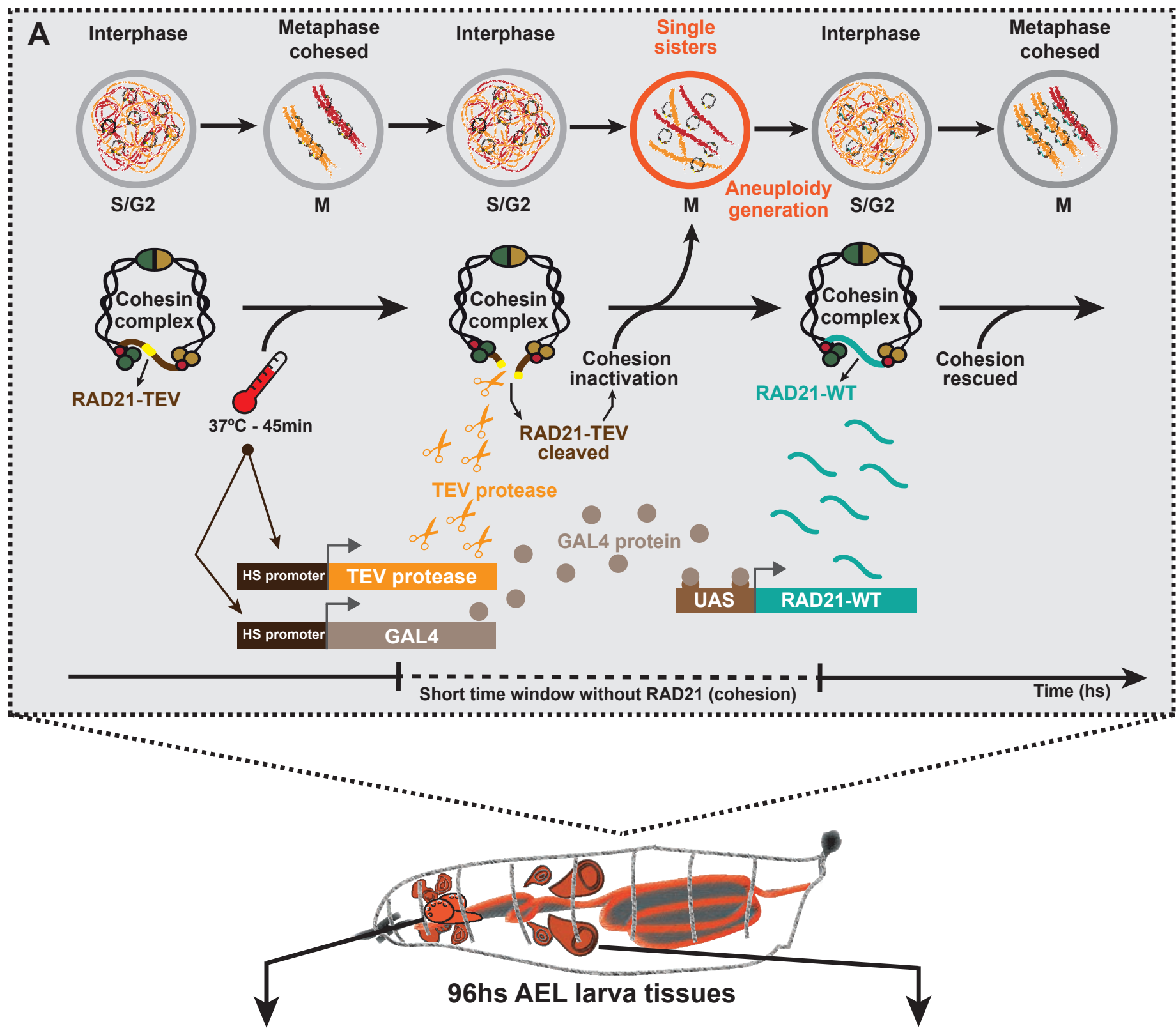

$A^{\prime}$

Brain

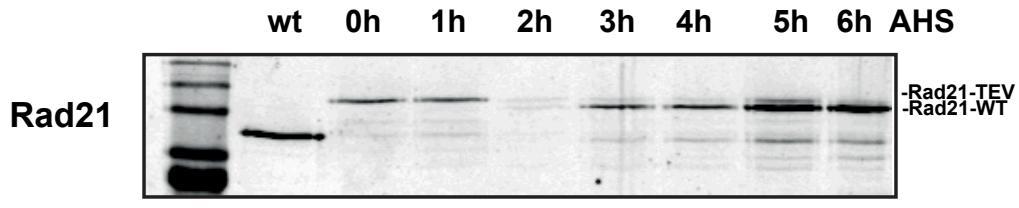

V5

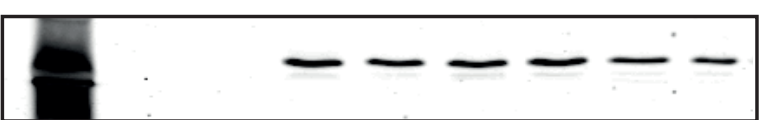

Tubulin

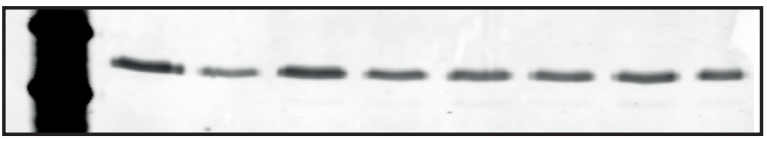

$A^{\prime \prime}$

Wing discs

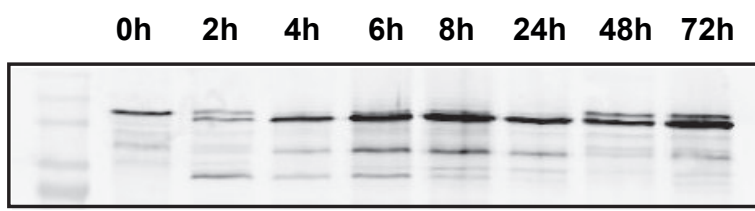

AHS

-Rad211-TEV
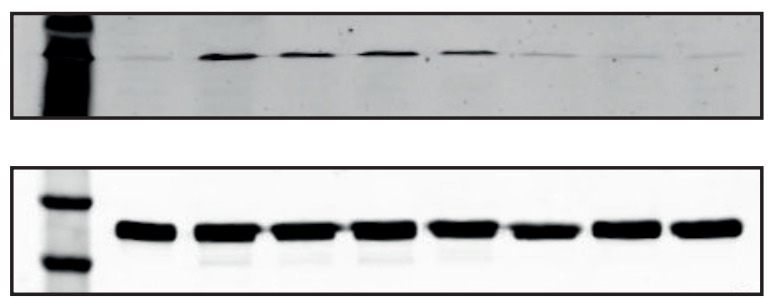
bioRxiv preprint doistips:tedojorg/10.1101/392746; this version posted August 15, 2018. The copyright holder for this preprint (which was

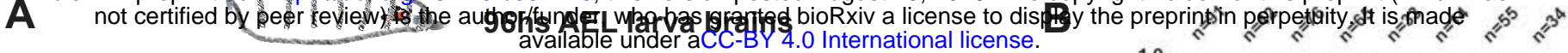
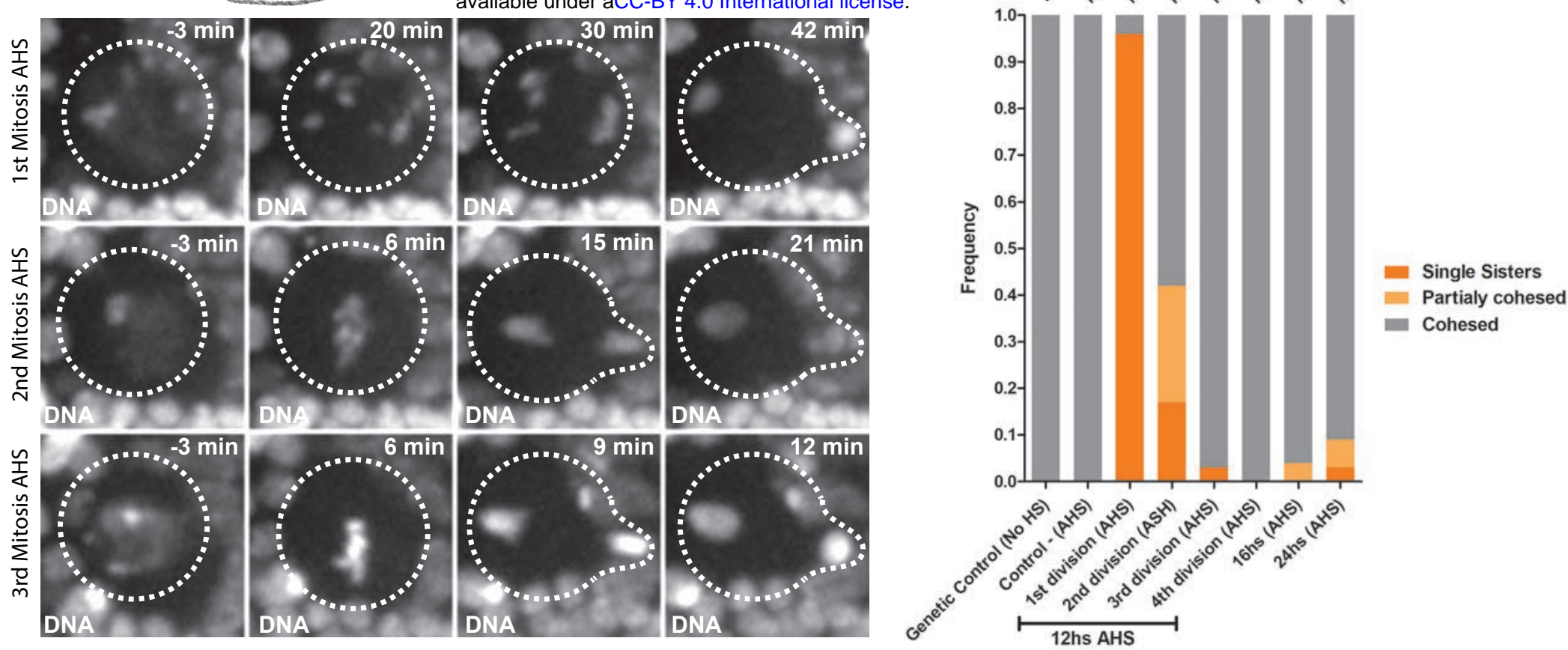

C

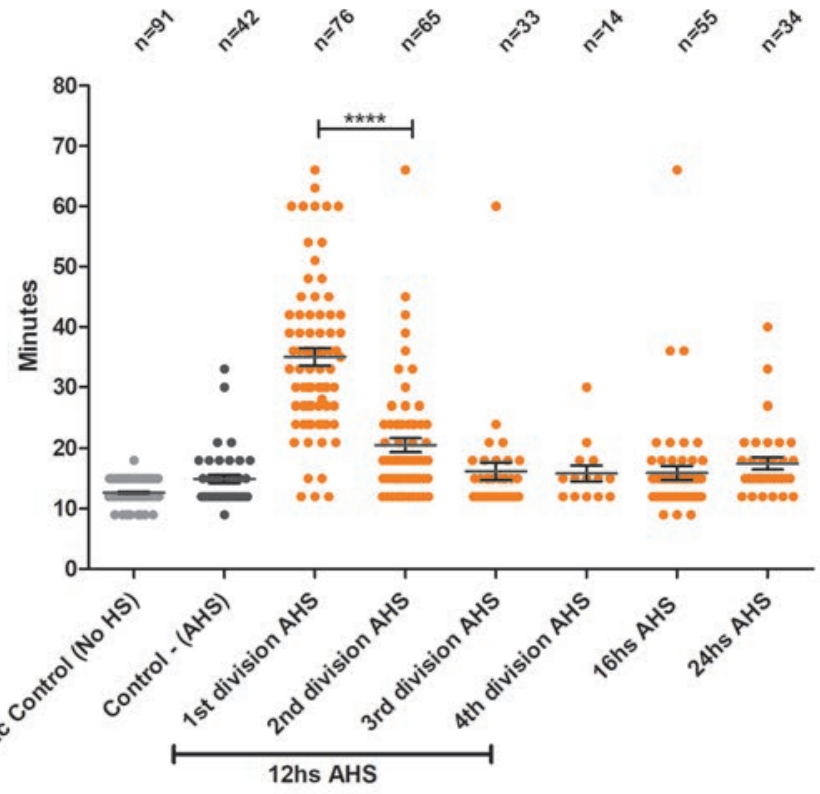

E

D

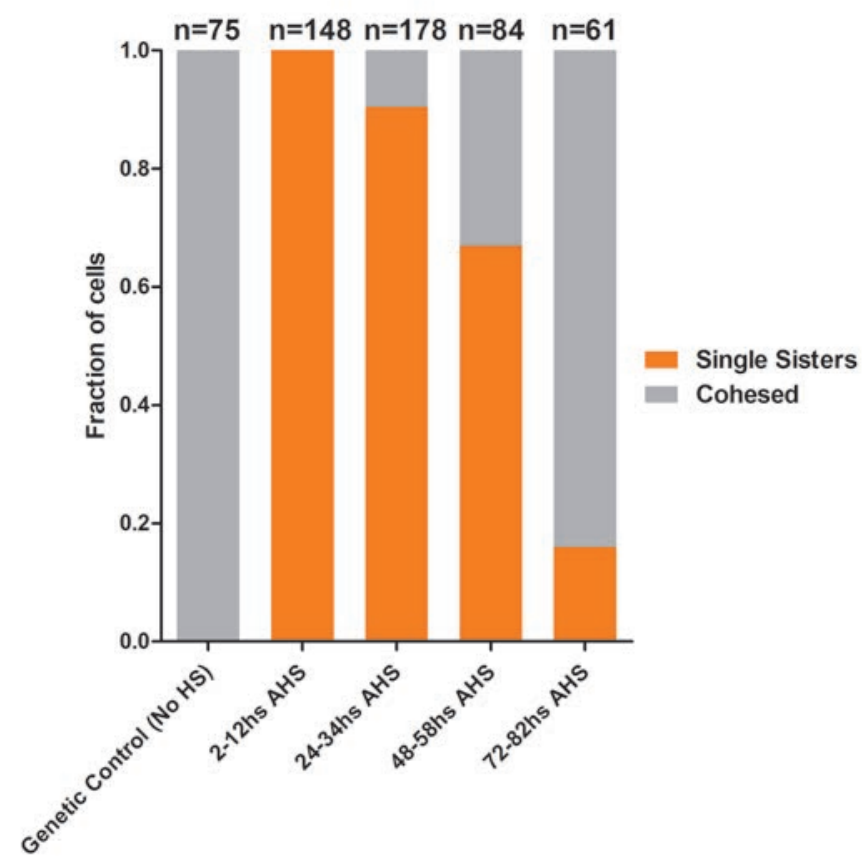

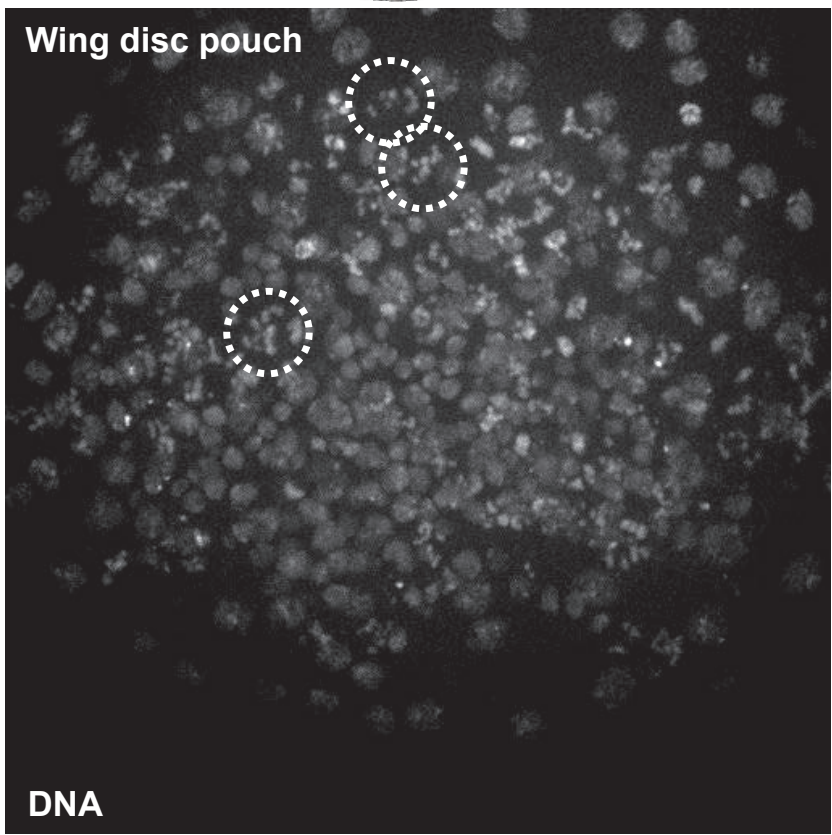

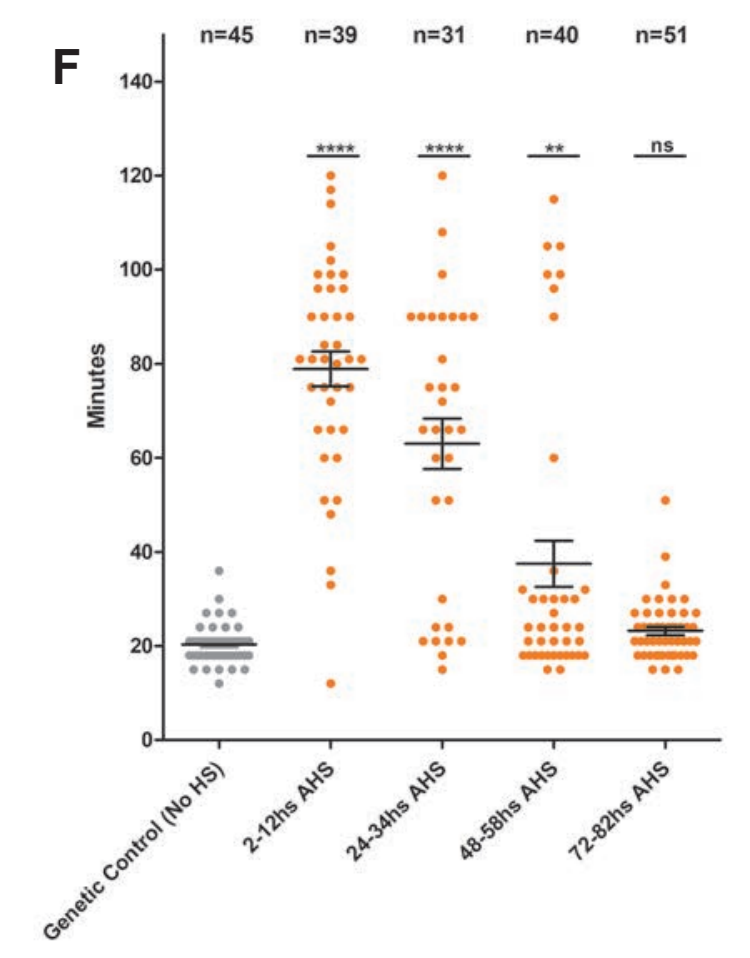

TISID 96hs AEL larva wing discs
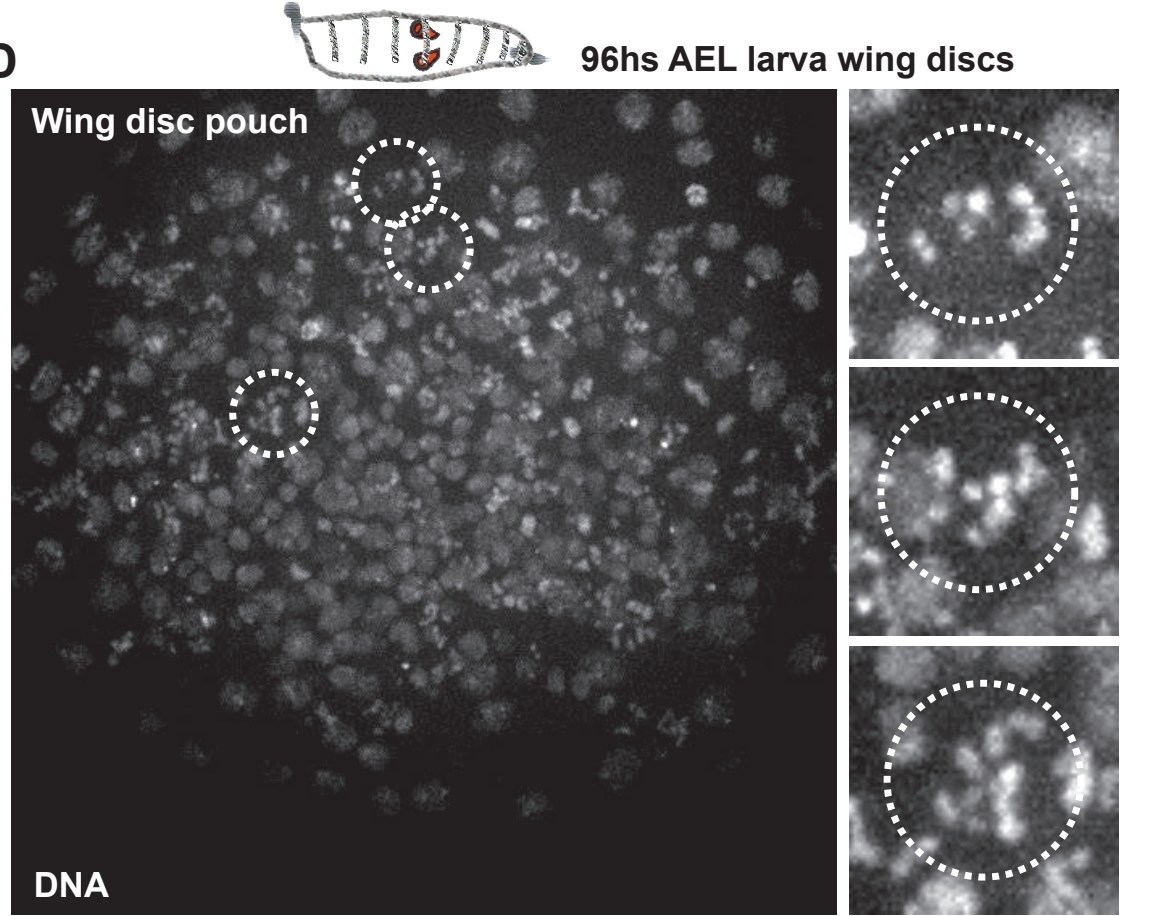

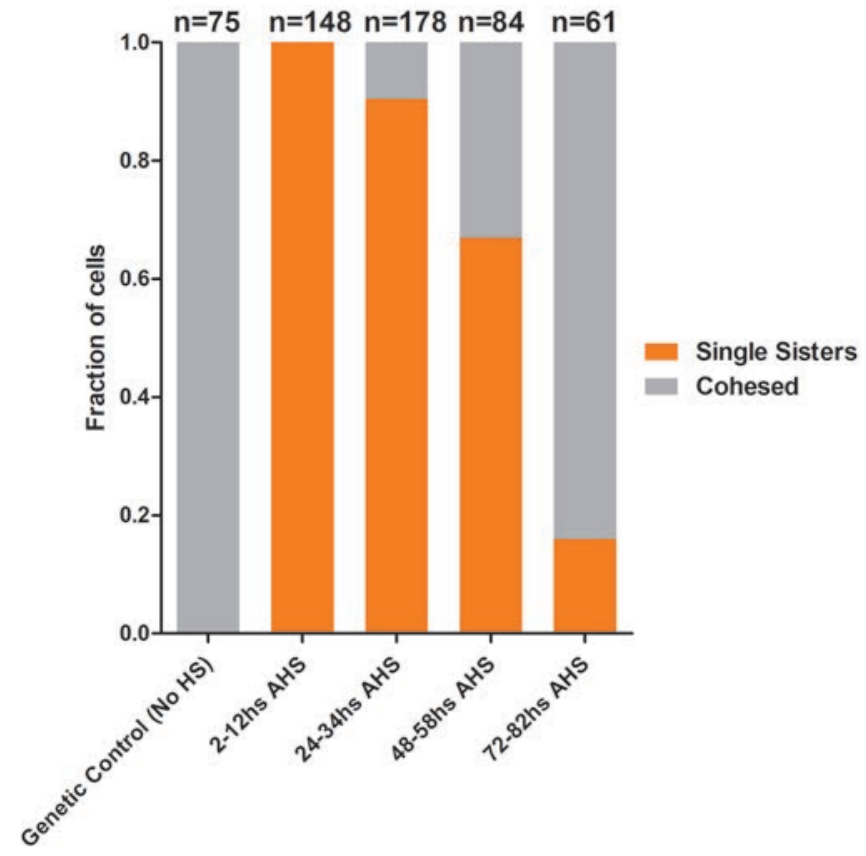




\section{Figure 3}
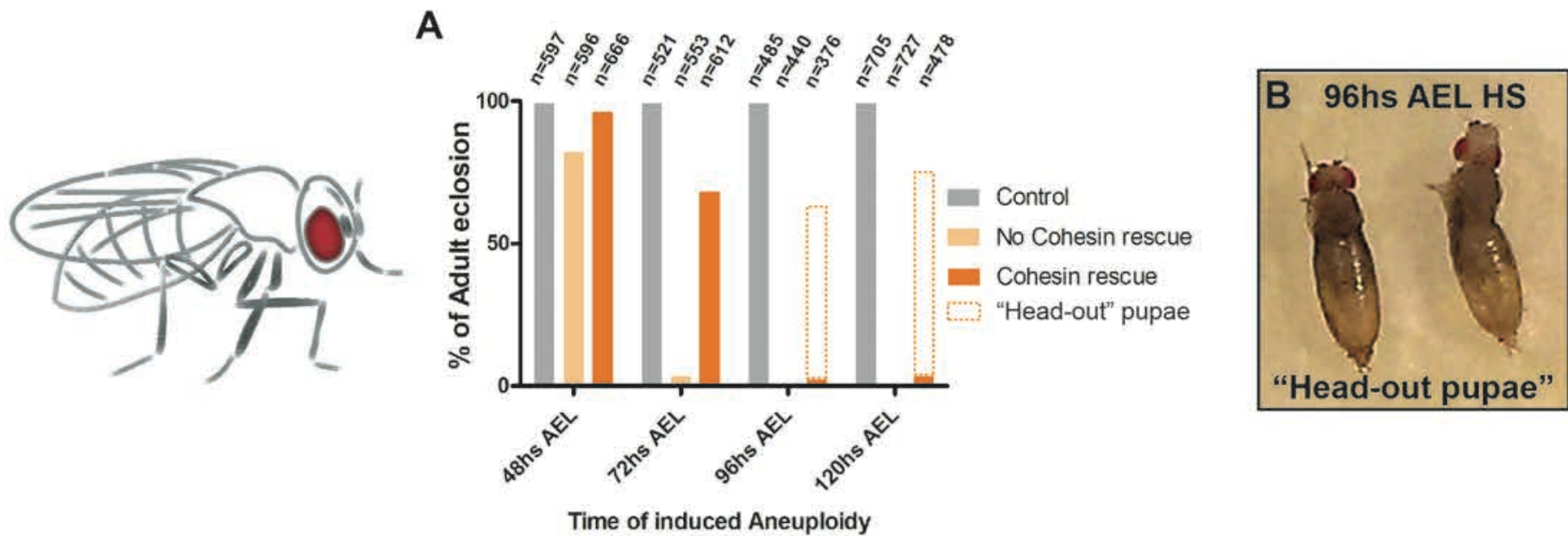

C
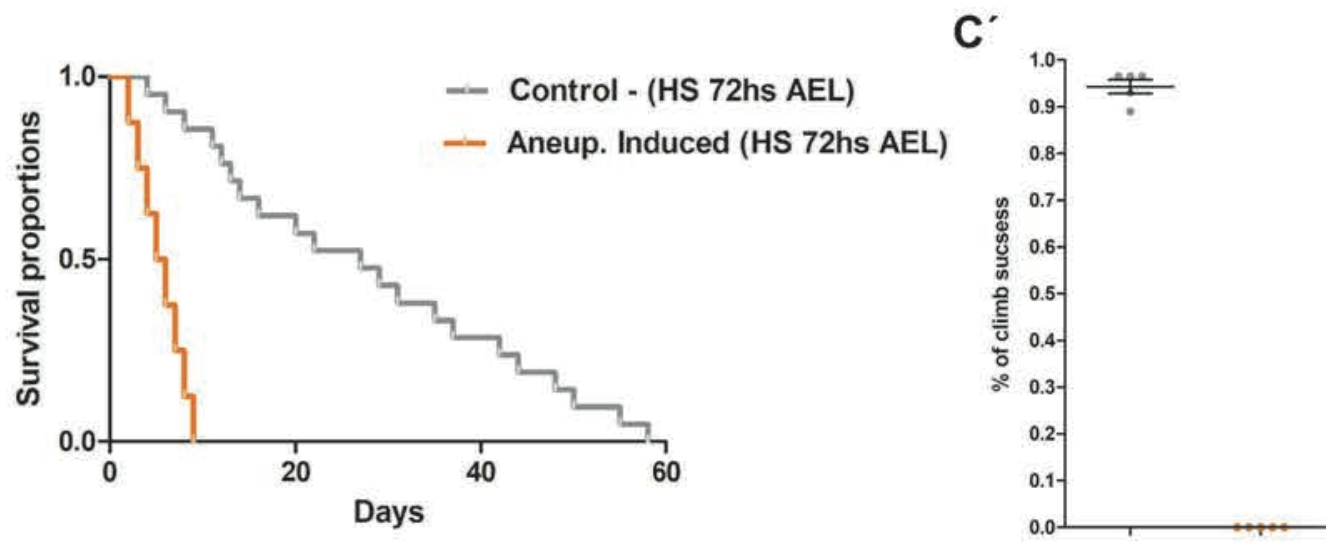
bioRxiv preprint doi: https://doi.org/10.1101/392746; this version posted August 15, 2018. The copyright holder for this preprint (which was

not certified by peer review) is the author/funder, who has granted bioRxiv a license to display the preprint in perpetuity. It is made available under aCC-BY 4.0 International license.

\section{Figure 4}

A
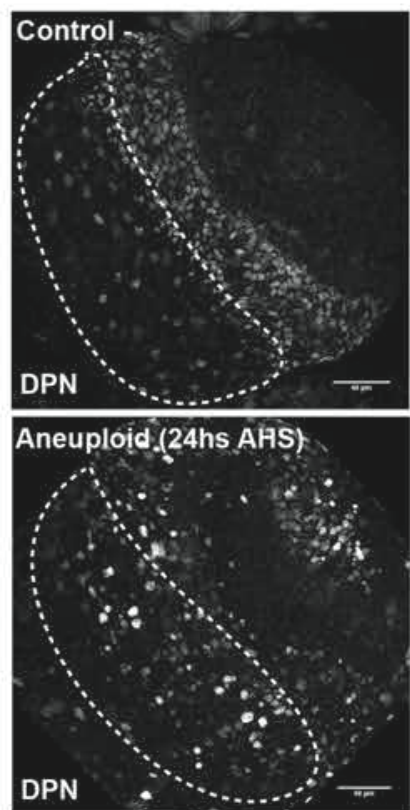

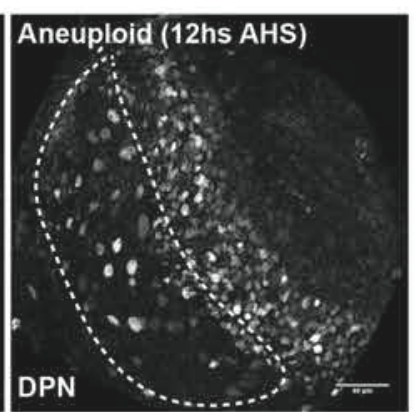

Aneuploid (48hs AHS)

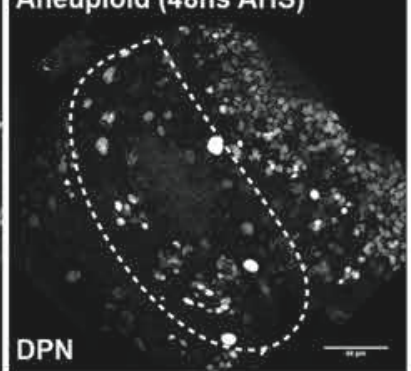

$A^{\prime}$

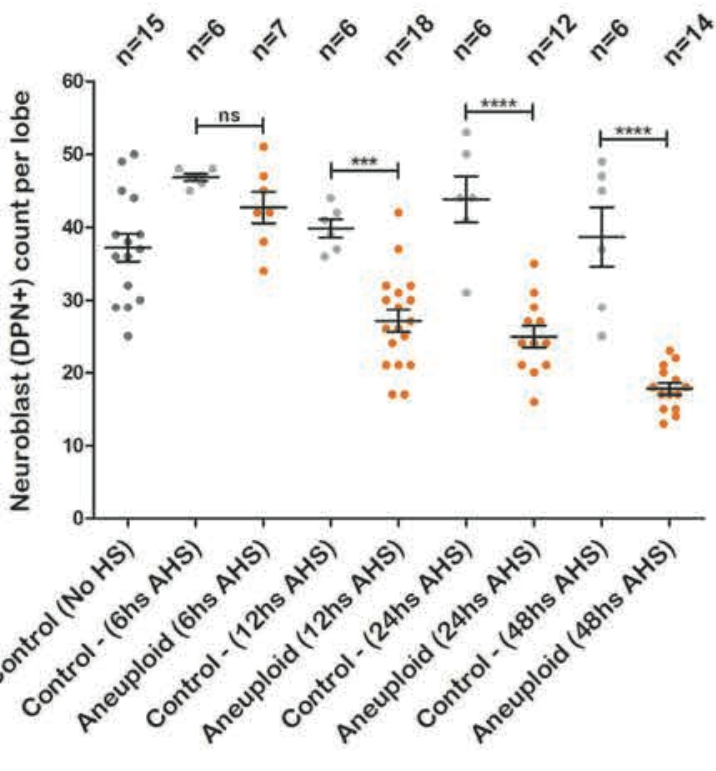

B

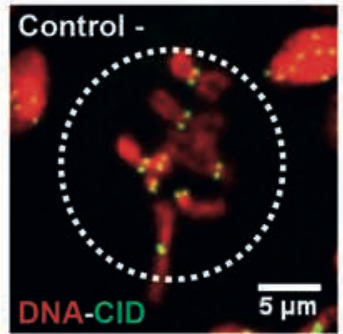

C

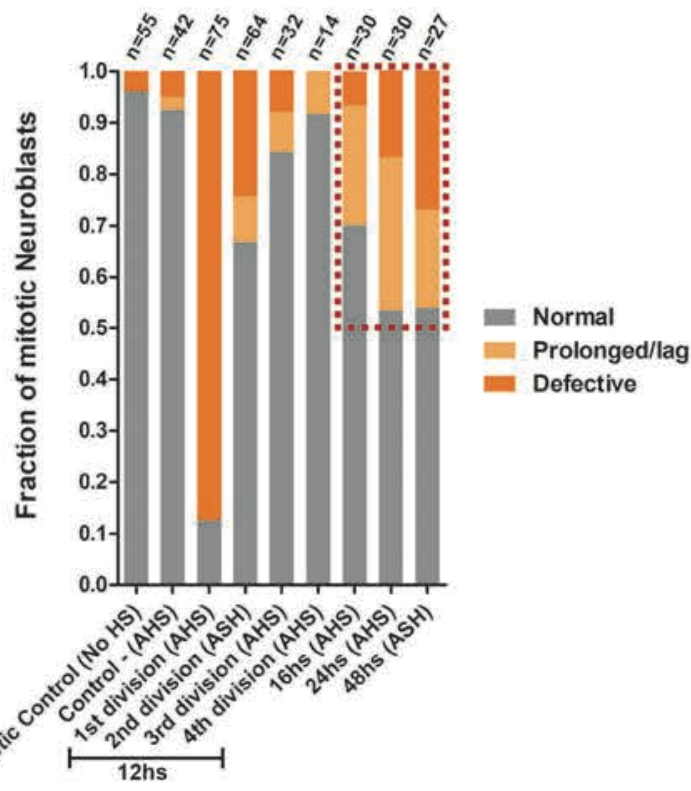

D".

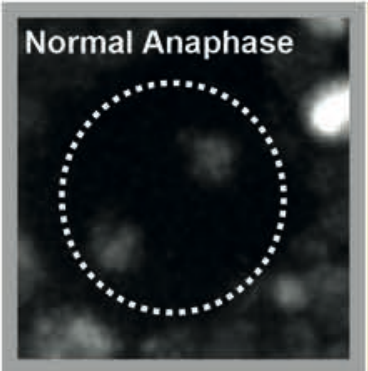

Aneuploid (24hs AHS) Aneuploid (24hs AHS)

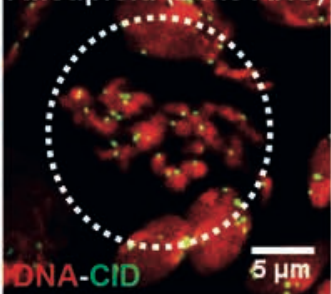

D $n=60$

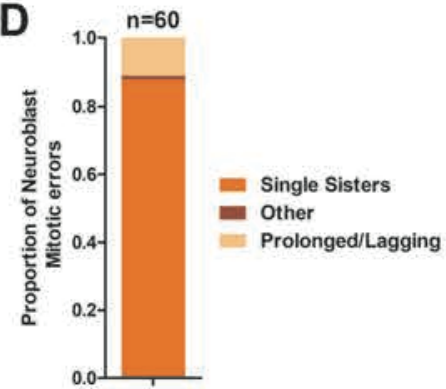

2-12hs after induced Aneuploidy

D'

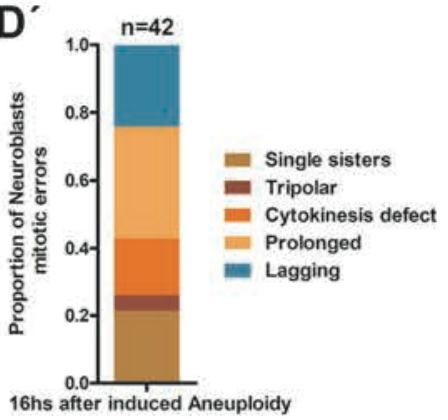

$16 \mathrm{hs}$ after induced Aneuploidy
B'

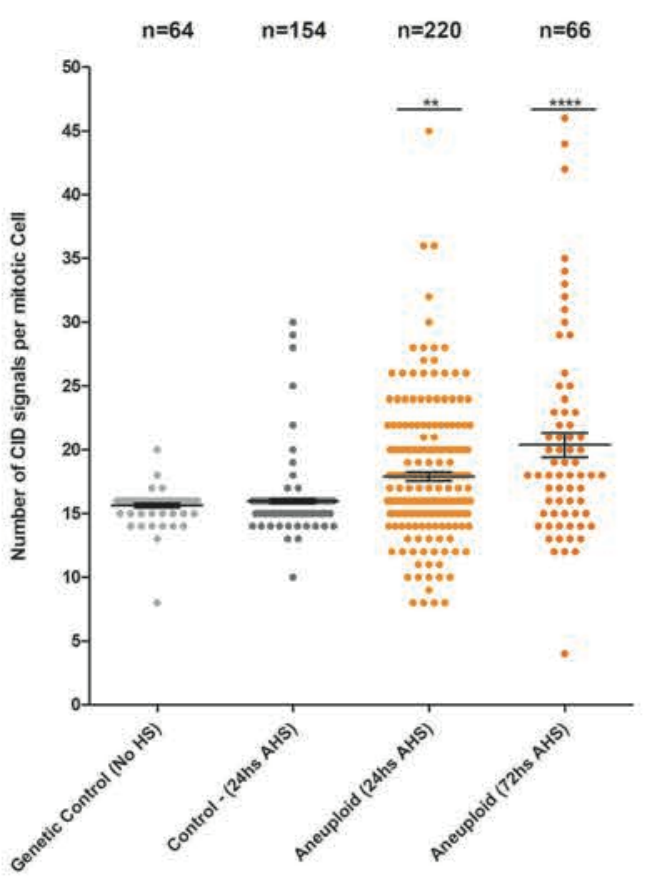

E

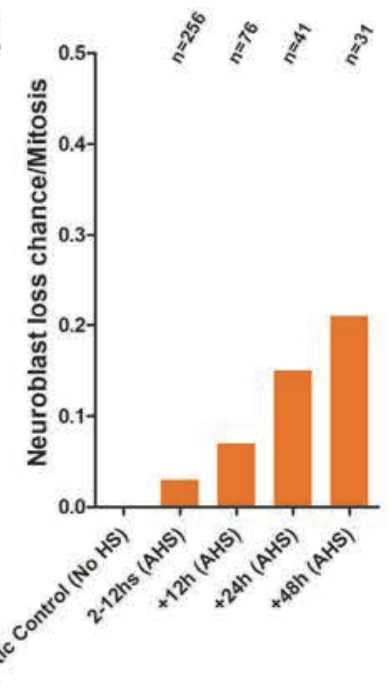




\section{Figure 5}

A

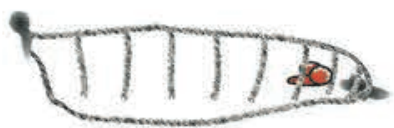

96hs AEL larva brains

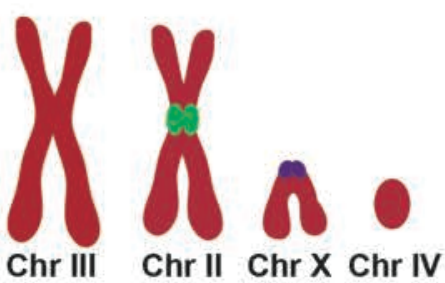

Chr III available under aCC-BY 4.0 International license.
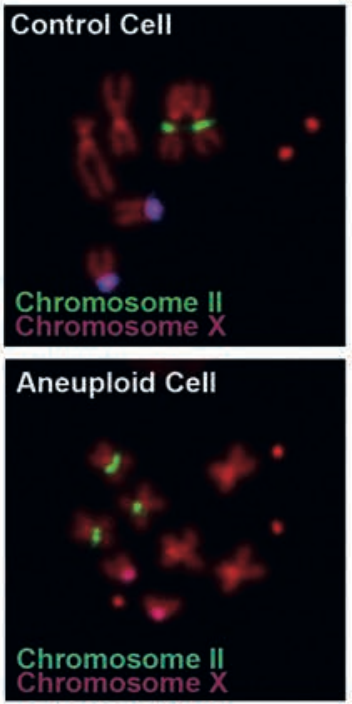

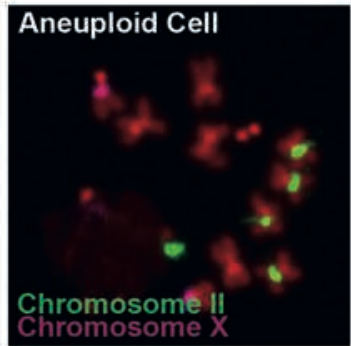

Aneuploid Cell

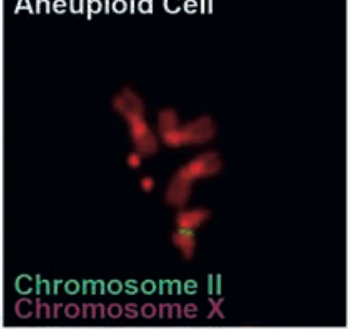

B

Theorethical model for indivudual chromatid segregation

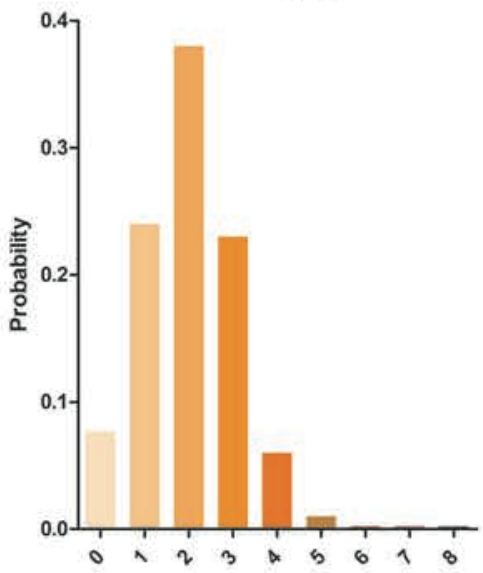

C
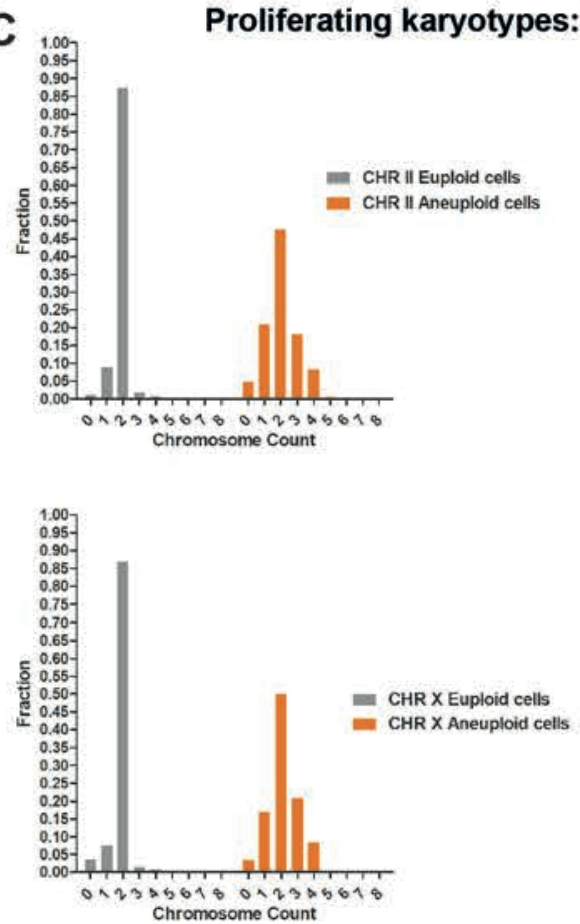

D
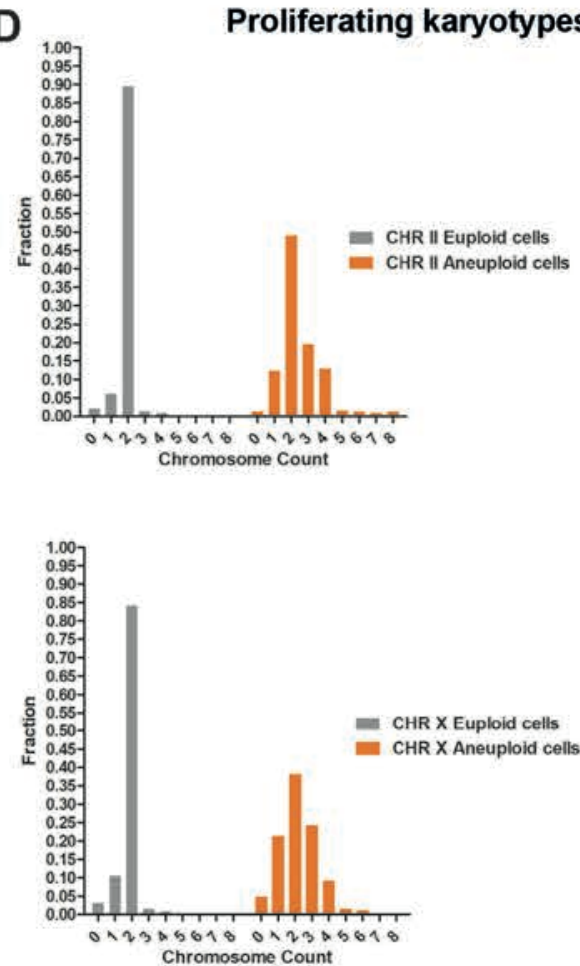
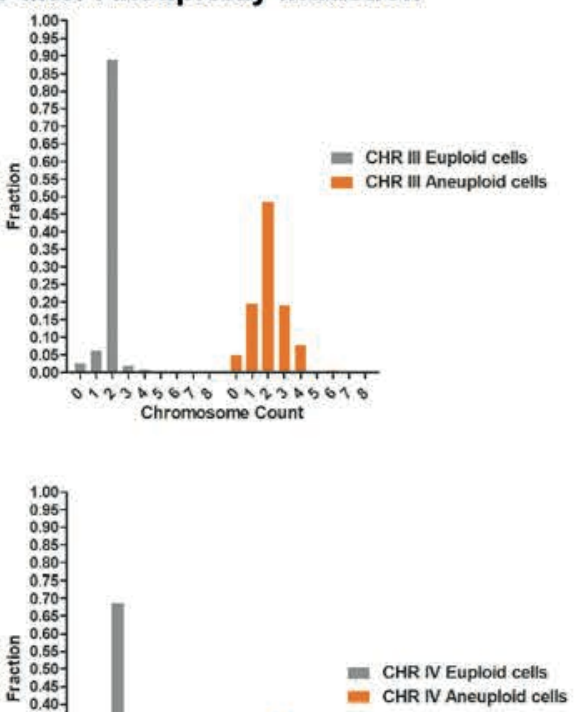

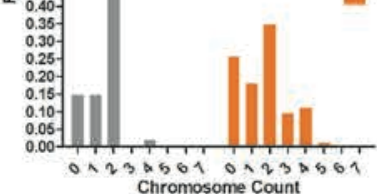

$C^{\prime}$

Total loss of chromosomes II,III or X: $\mathbf{8 h s}$ after Aneuploidy induction
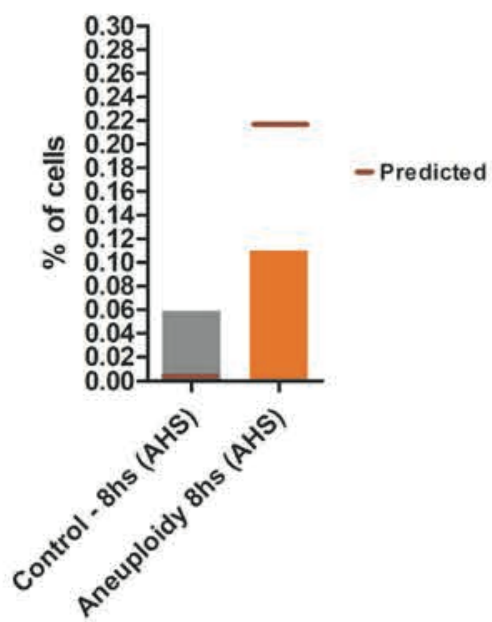

D'

Total loss of chromosomes II,III or X: 24hs after Aneuploidy induction

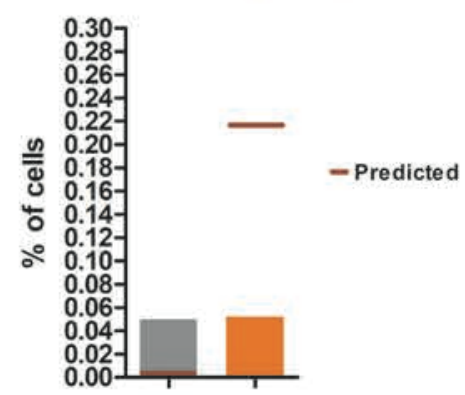

CHR IV Euploid cells - CHR N Aneuploid cells

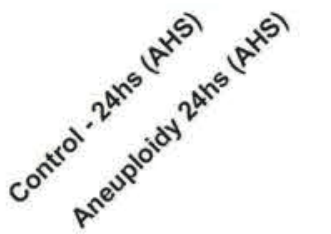


bioRxiv preprint doi: https://doi.org/10.1101/392746; this version posted August 15, 2018. The copyright holder for this preprint (which was

not certified by peer review) is the author/funder, who has granted bioRxiv a license to display the preprint in perpetuity. It is made available under aCC-BY 4.0 International license.

\section{Figure 6}
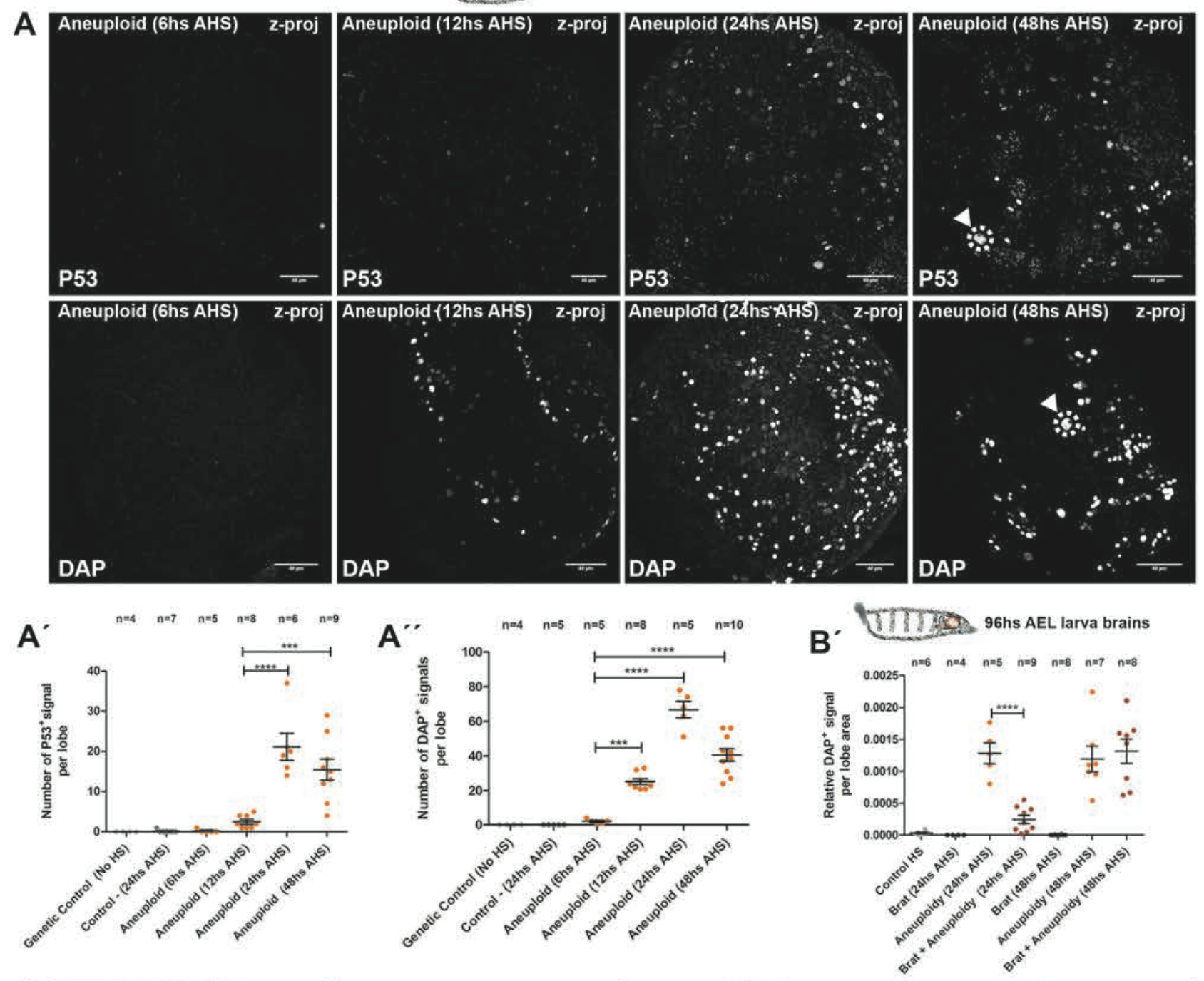

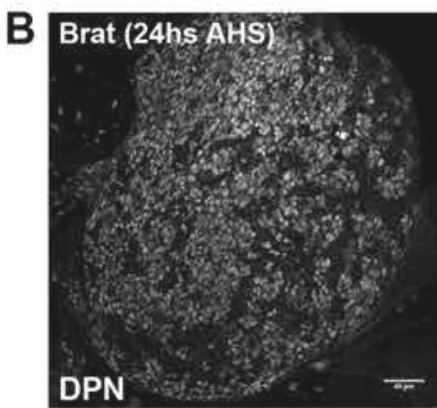

Aneuploidy (24hs AHS)

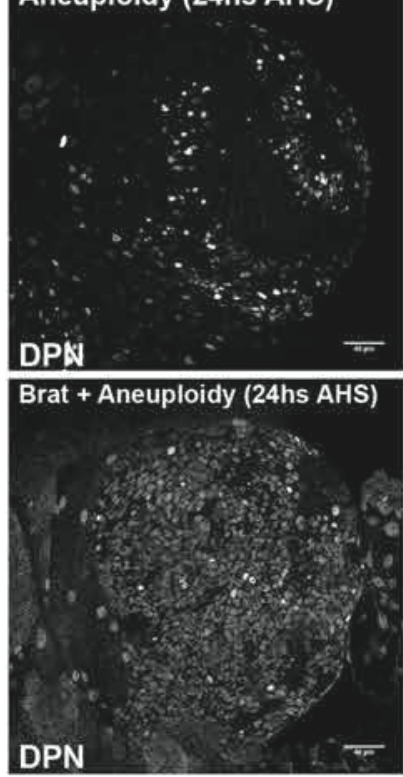

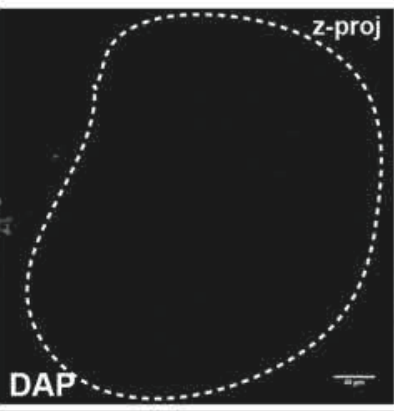

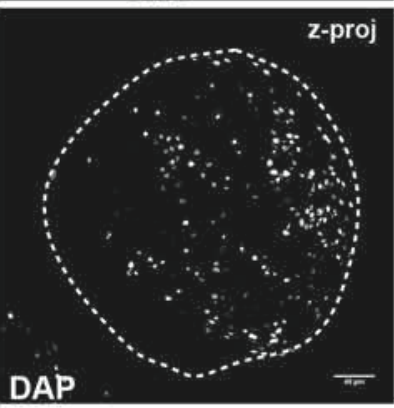

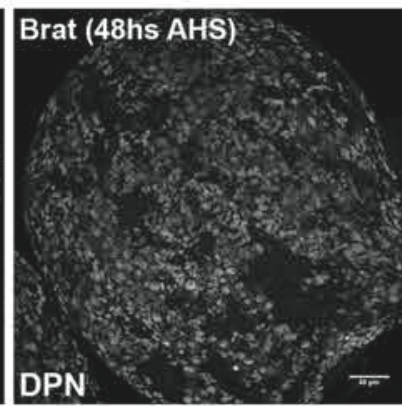

Aneuploidy (48hs AHS)

DAP

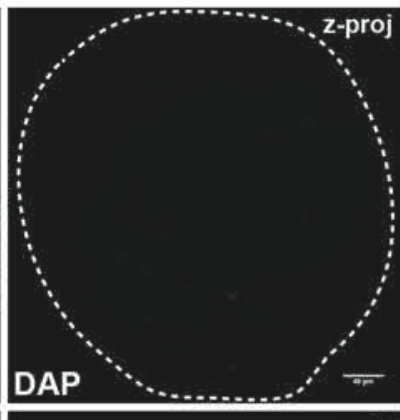

z-proj<smiles></smiles>

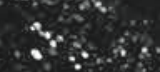

DPNं

DAP

Brat + Aneuploidy (48hs AHS)

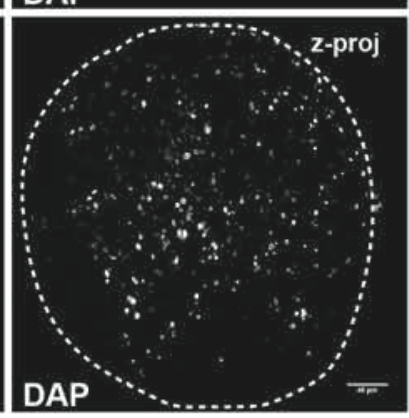


$\mathbf{A}$
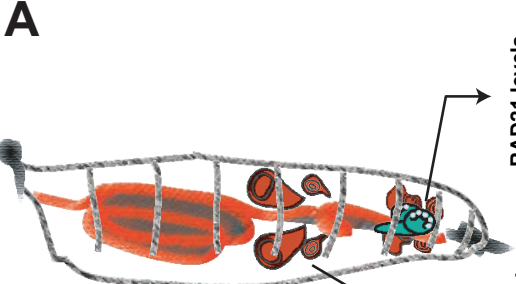

96hs AEL larva tissues
B

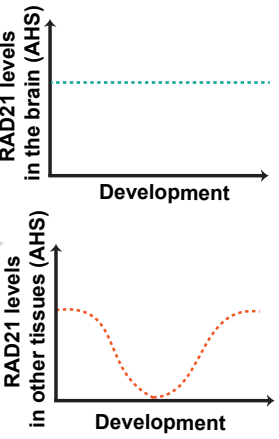

Myc

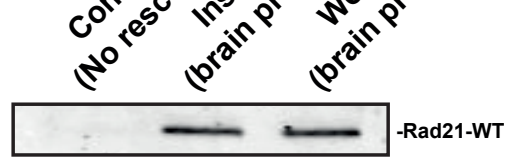

Rad21

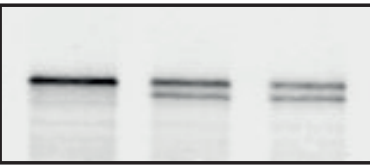
-Rad21-TEV -Rad21-WT

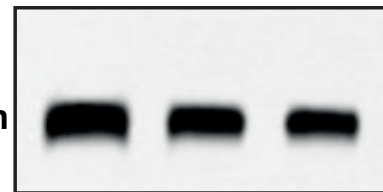

C

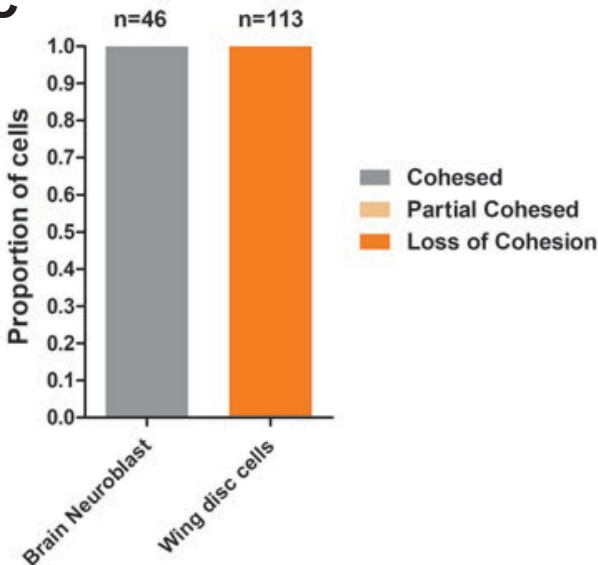

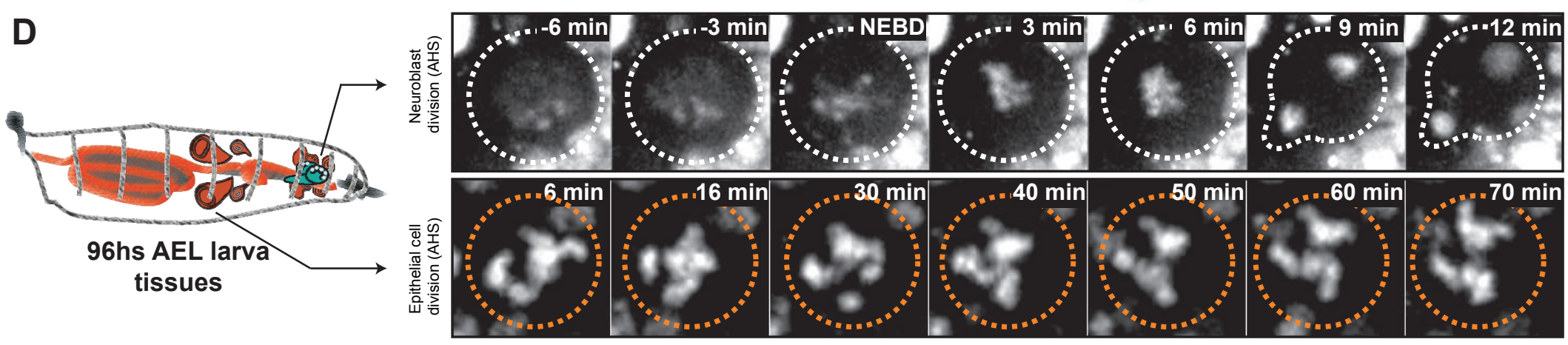
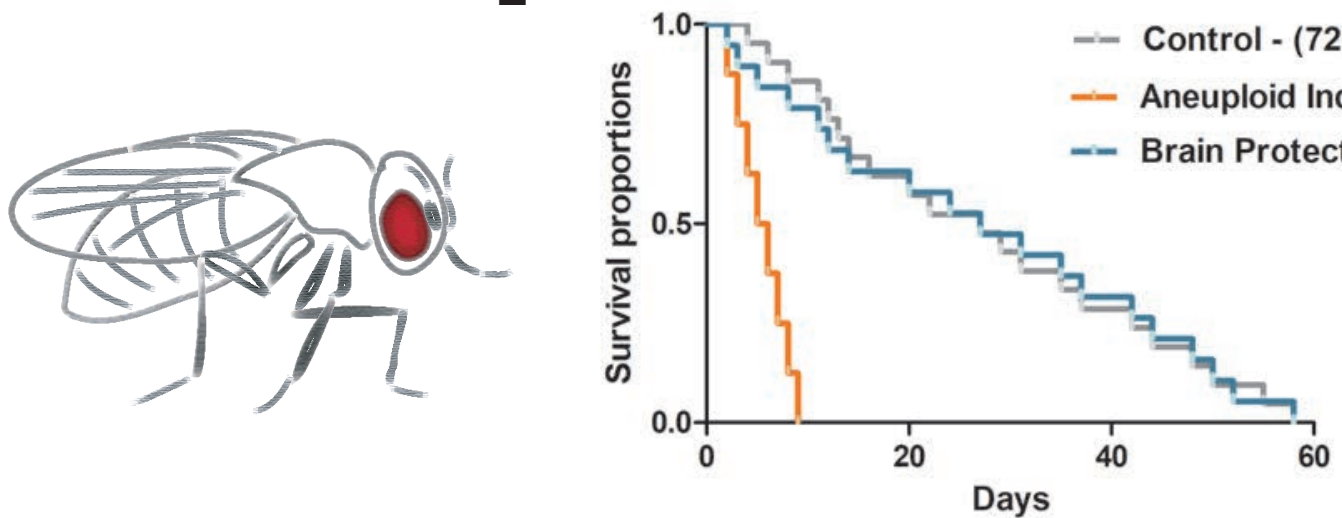

E

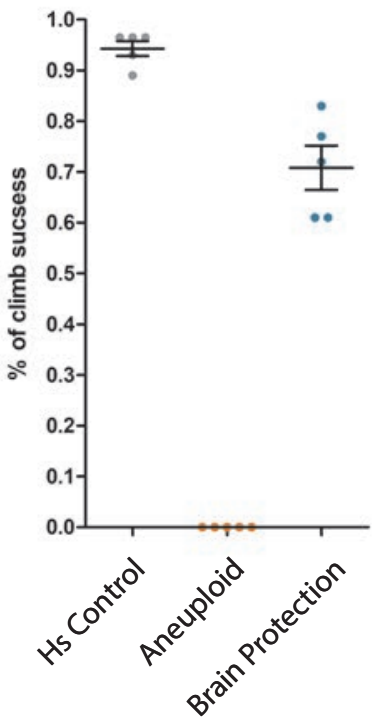




\section{Supplementary Figures:}

Supplementary Figure 1

\section{Heat-shock treatment induces brain aneuploidy at all stages of development.}

A to $\mathbf{A}^{\prime \prime}-$ Stills from live imaging of lobe brains at different larvae stages $(48,72$ and $96 \mathrm{hs}$ AEL); dashed circles are highlighting the $\mathrm{Nbs}$ in the lobes $(\mathrm{N}>3$ brains per condition). The number of dividing Nbs increase with larvae development. Cohesive state of Nbs after the loss of cohesion and subsequent rescue in 48, 72 and 96hs AEL larvae were plotted.

B- Western blot of RAD21 cleavage and rescue dynamics in 72hs AEL larvae brains (over 10 western blots were performed to validate the system).

\section{Supplementary Figure 2}

Epithelial tissues recover from high levels of aneuploidy by cell death and compensatory proliferation.

A to $\mathbf{A}^{\prime}$ : A- Reversible cohesin cleavage results in apoptosis in the $3^{\text {rd }}$ instar wing discs (dashed shapes depict the wing disc areas). The amount of apoptosis per disc was measured by area of cleaved caspase 3 (CC3) immunofluorescence at 24, 48 and 72hs AHS. A'- Rescue of cohesin function significantly reduced the amount of apoptosis within $48 \mathrm{hs}$ after of the heat shock.

In contrast, chronic inactivation of cohesin complex (No cohesin rescue) displayed high levels of apoptosis through time. Control- (Control HS); Control+ (Irradiation: 4,000 rads); BHS (Before Heat-Shock, genetic control); AHS (After Heat-Shock, condition); BI (Before Irradiation); AI (After Irradiation). ${ }^{*}=\mathrm{P}<0.05 ; * * *=\mathrm{P}<0.0001$. Scale bar $=40 \mu \mathrm{m}$. z-proj (z projection).

\section{Supplementary Figure 3}

\section{RAD21 cleavage and rescue induces loss of cohesion in all examined dividing tissues}

A- Stills from live imaging of leg, eye, antennae and halter $3^{\text {rd }}$ instar imaginal discs after induction of RAD21 cleavage. Dashed squares display epithelial cells from the imaginal discs undergoing mitosis with loss of cohesion (see enlarged picture).

B- The cell cycle profile evaluation of the $3^{\text {rd }}$ instar control wing disc with or without the heat shock, using the FLY-FUCCI system. The high incidence of cells affected by reversible cohesin cleavage is consistent with a high frequency of cells in G2/M in this tissue (see Merge). GFP: G1 cells; RFP: S phase cells; Merge: G2/M Cells ( $n>500$, at least three wing discs analyzed)

\section{Supplementary Figure 4}

Aneuploidy results in low frequency of stem identity loss and cell death in Neuroblasts.

A to $\mathbf{A}^{\prime}$ : A- Pictures from fixed samples of $3^{\text {rd }}$ instar larvae lobe brains stained with Deadpan (DPN), Prospero (Pros) and Histone RFP (DNA). Induction of aneuploidy results in the loss of 
stem cell identity measured by the absent of Deadpan (stem cell marker, white arrowhead with dashed circle), appearance of Prospero (differentiation marker, yellow arrowhead with dashed circle) or both markers together in cell nucleus with "Nbs shape like". A'- Percentage of loss of stem cell identity in the neural stem cell pool at different time points after the induction of aneuploidy. These events are observed at very low frequency. $n=$ number of Nbs like cells. Scale bar $=40 \mu \mathrm{m}$

B to $\mathbf{B}^{\prime \prime}$ : B- Pictures from fixed samples of $3^{\text {rd }}$ instar larvae lobe brains stained with Deadpan (DPN), Cleaved Caspase 3 (CC3, death marker), DCP1 (death marker) and rhodamine phalloidin (Actin). Induction of aneuploidy results in cell death measured by the presence of CC3 or DCP1 signals (white arrowheads with dashed circles) in cells with "Nbs shape like". B' and B"- Quantification of cell death signals CC3 and DCP1 per larvae brain lobes at 24hs AHS. The presence of positive signal for the cell death markers in Nbs shape like cells is very low. $* *=\mathrm{P}<0.01$. ns $=$ not significant. Scale bar $=40 \mu \mathrm{m}$. z-proj (z projection)

C- Quantification of Nbs at the central brain in $3^{\text {rd }}$ instar lobe brains assessed by immunofluorescence with the Nbs marker DPN. Inhibition of apoptosis by over-expression of baculovirus P35 does not rescue Nbs number after 24hs induced aneuploidy. $\mathrm{n}=$ number of lobe brains. $* * * *=\mathrm{P}<0.0001$. $\mathrm{ns}=$ not significant.

\section{Supplementary Figure 5}

Aneuploidy induced stress response is particularly delayed in the Neuroblasts

A to $\mathbf{A}^{\prime \prime}$ : Pictures from fixed samples of $3^{\text {rd }}$ instar larvae lobe brains showing the immunofluorescence of canonical stress response markers P53 and Dacapo (DAP) together with the Nbs marker (DPN) at $48 \mathrm{hs}$ AHS. Nbs display a delayed aneuploidy stress response at $48 \mathrm{hs}$ AHS (arrowheads with dashed circles). $\mathbf{A}^{\prime}$ and $\mathbf{A}^{\prime \prime}$ - Quantification of the kinetics of the aneuploidy induced stress response at $6,12,24$, and $48 \mathrm{hs}$ AHS in Nbs (DPN+). Scale bar $=$ $40 \mu \mathrm{m}$.

\section{Supplementary Figure 6}

Aneuploidy induced P53 accumulation is delayed in the neural stem cell pool.

A to $\mathbf{A}^{\prime}$ : A- Brat mutant lobe brains showed a clear reduction in the presence of the aneuploidy induced stress marker P53 at 24hs AHS. A'- Quantification of relative P53 positive signal per lobe area from 24 to $48 \mathrm{hs}$ AHS. $\mathrm{n}=$ number of lobe brains. $* * * *=\mathrm{P}<0.0001$. Scale bar $=40 \mu \mathrm{m}$. z-proj (z projection).

\section{Supplementary Figure 7}

Adult brains do not show any significant alteration in size after acute induction of aneuploidy during development. 
A to $\mathbf{A}^{\prime \prime}$ : A- Dissected brains of adult flies from a control and a developmental aneuploidyinduced (72hs AEL heat-shock) organisms. $\mathbf{A}^{\prime}$ and $\mathbf{A}^{\prime \prime}$ - Quantifications of lobe diameter and brain length in control and developmental aneuploidy-induced (72 and 96hs AEL heat-shock) adult flies showed no significant differences. $n=$ number brains. $n s=$ no significant

B- Histology analysis of brains from control and aneuploidy-induced during development (72hs AEL heat-shock) adult flies, one day after eclosion. Frontal sections at approximately midbrain showed no signal of neurodegenerative process (vacuolization). H\&E= Hematoxylin and Eosin.

\section{\#Supplementary Movies:}

Supplementary Movie 1: Demonstration of cohesin cleavage and rescue system in a single Neuroblast after the heat shock.

Movie depicting live imaging of the first and second mitosis after the heat chock, in a single $3^{\text {rd }}$ instar larvae Neuroblast; Imaging of HisH2AvD mRFP1 in 3 minute timeframes. First division after the heat shock results in complete cohesin loss, while the second provides a full cohesin rescue.

Supplementary Movie 2: Demonstration of cohesin cleavage and rescue in the entire larval brain lobe after the heat shock.

Movie depicting live imaging of the $3^{\text {rd }}$ instar larvae brain lobe; Imaging of HisH2AvD mRFP1 in 3 minute timeframes. First divisions after the heat shock result in complete cohesin loss and genome shuffling, consecutive divisions result in cohesin rescue.

Supplementary Movie 3: Cohesin loss and rescue results in eclosion of adults with motion defects, despite healthy wing and appendage morphology.

Movie depicting live imaging of the adult eclosed after the aneuploidy challenge at the age of $72 \mathrm{hs}$ after egg laying.

Supplementary Movie 4: A Climbing assay comparing heat shock controls and flies challenged with aneuploidy during development.

Supplementary Movie 5: 48hs AEL Heat-Shock results in eclosion of adults with lethargic behavior despite healthy wing and appendage morphology.

Movie depicting live imaging of the adult eclosed after the aneuploidy challenge at the age of 48hs after egg laying

Supplementary Movie 6: Climbing assay comparing brain-rescued flies and flies challenged with aneuploidy during development. 


\section{Supp Figure 1}

A
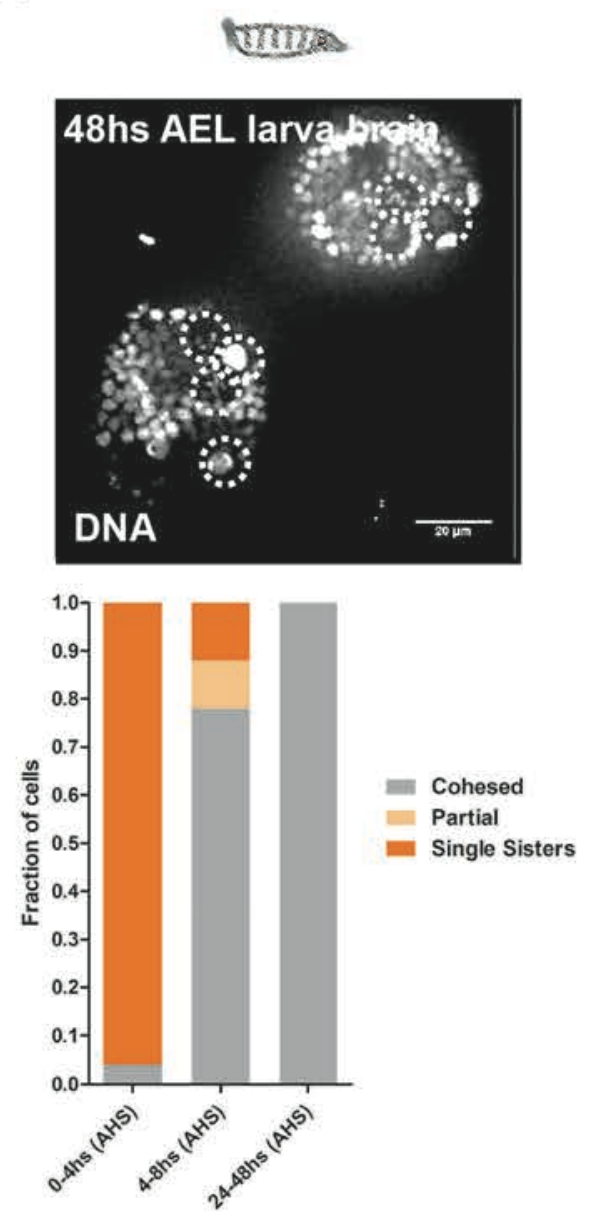

\section{$A^{\cdot}$}
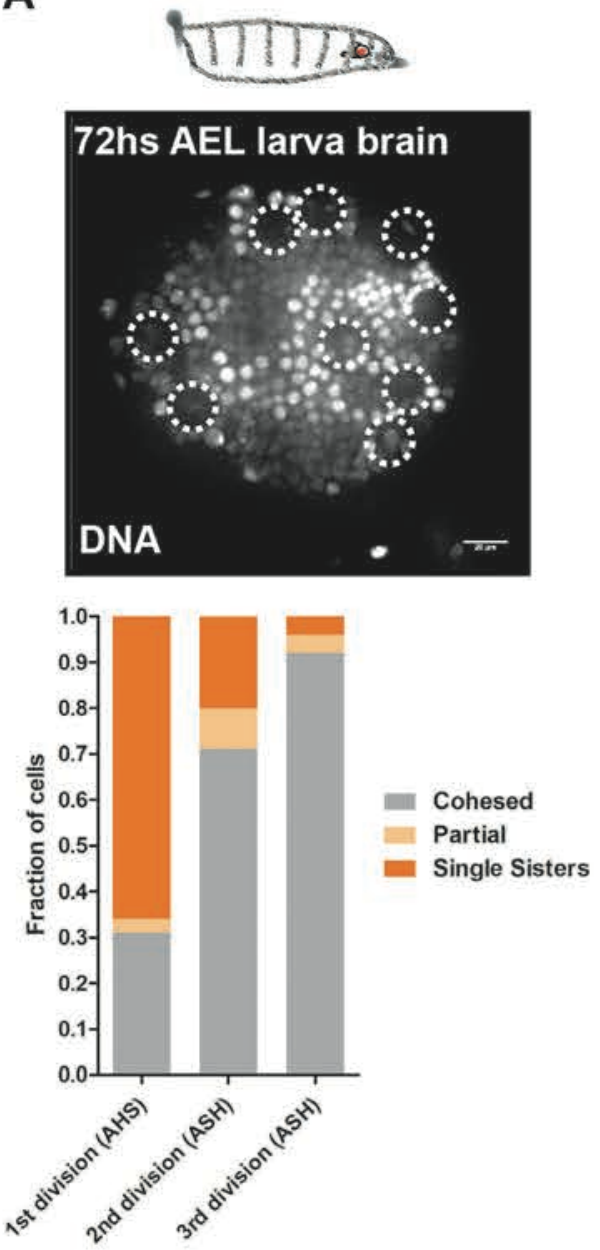
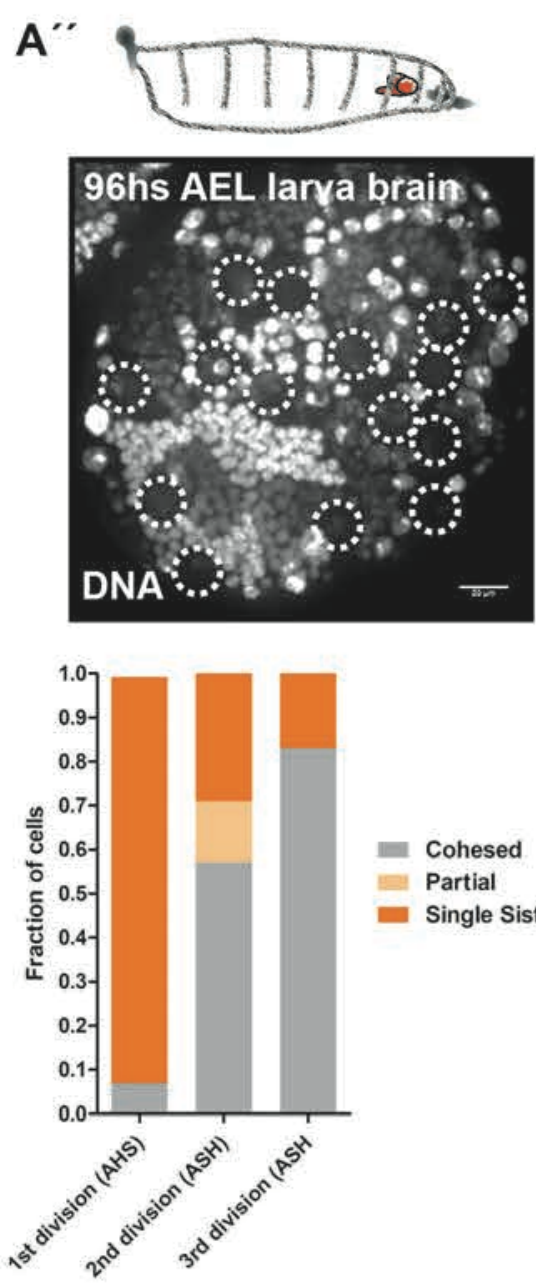

Cohesed

rartial

- Single Sisters

B

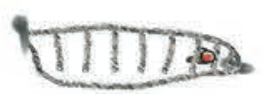

72hs AEL larva brains

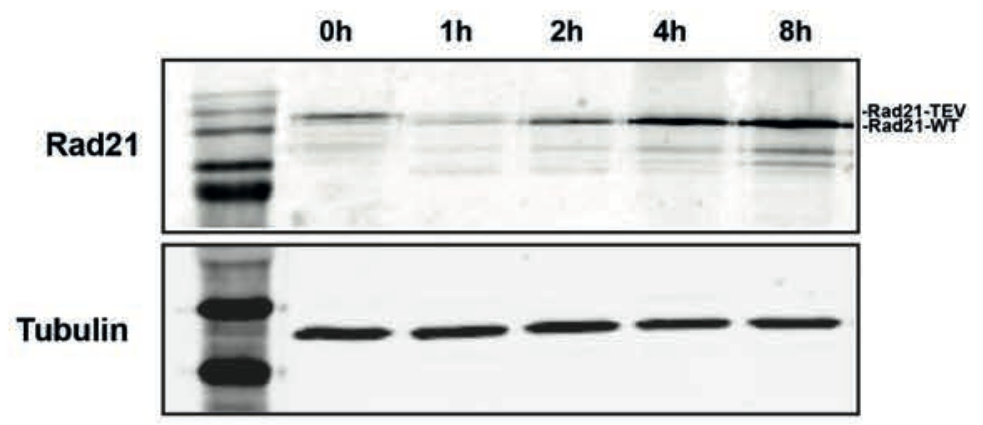




\section{Supp Figure 2}

A

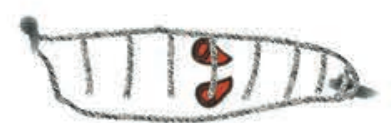

96hs AEL larva

wing discs
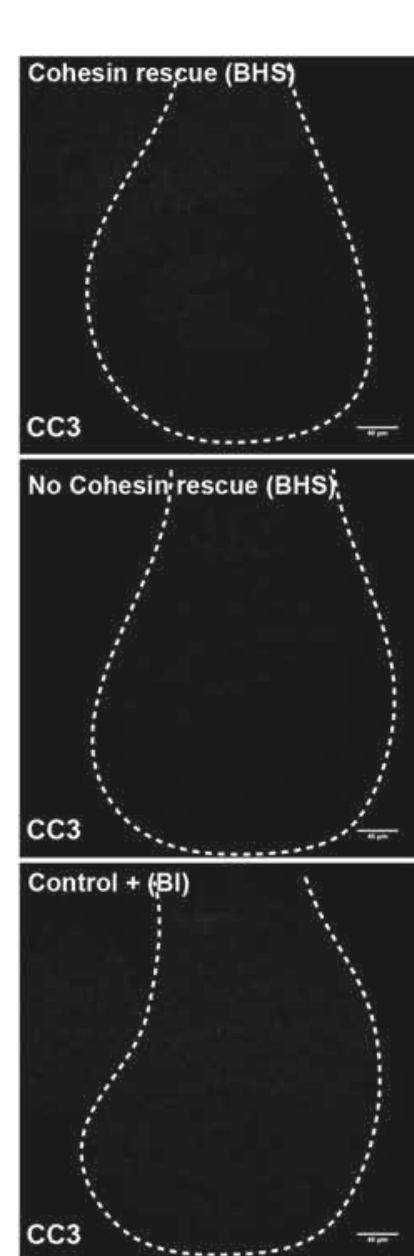

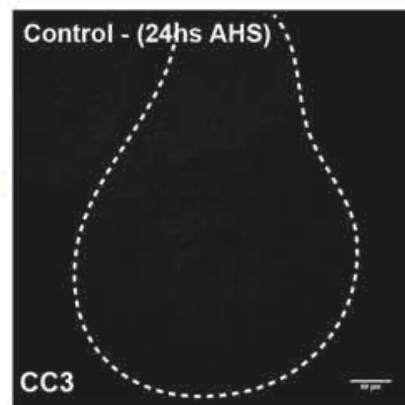

Cohesin rescue (24hs AHS)

CC3

No Cohesin rescue (24hs AHS)

CC3

Control + (24hs Al)

CC3

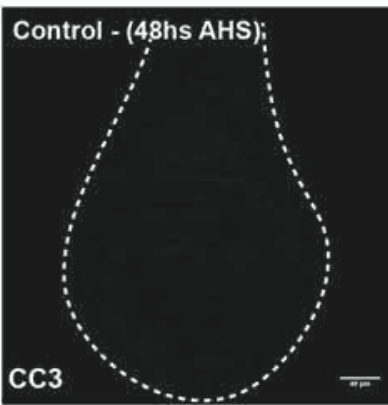

Cohesin rescue (48hs AHS)

CC3

No Cohesin rescue (48hs, AHS)

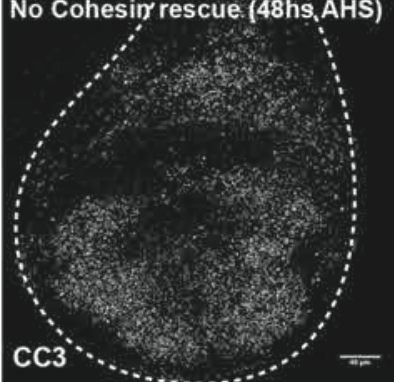

Control + (48hs Al)

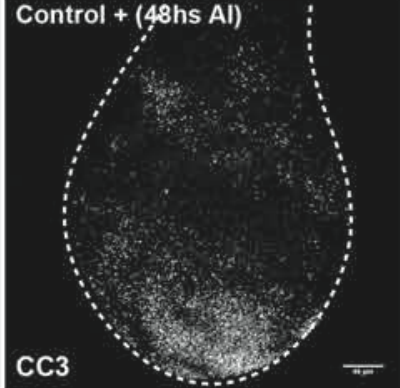

Control - i(72hs AHS)

CC3

Cohesin rescue (72hs AHS)

$\mathrm{CC} 3$

No Cohesin réscue (72̧hs AHS)

\section{CC3}

Control it (72hs Al)

CC3

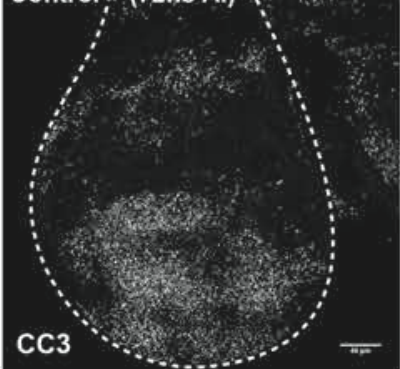

Time

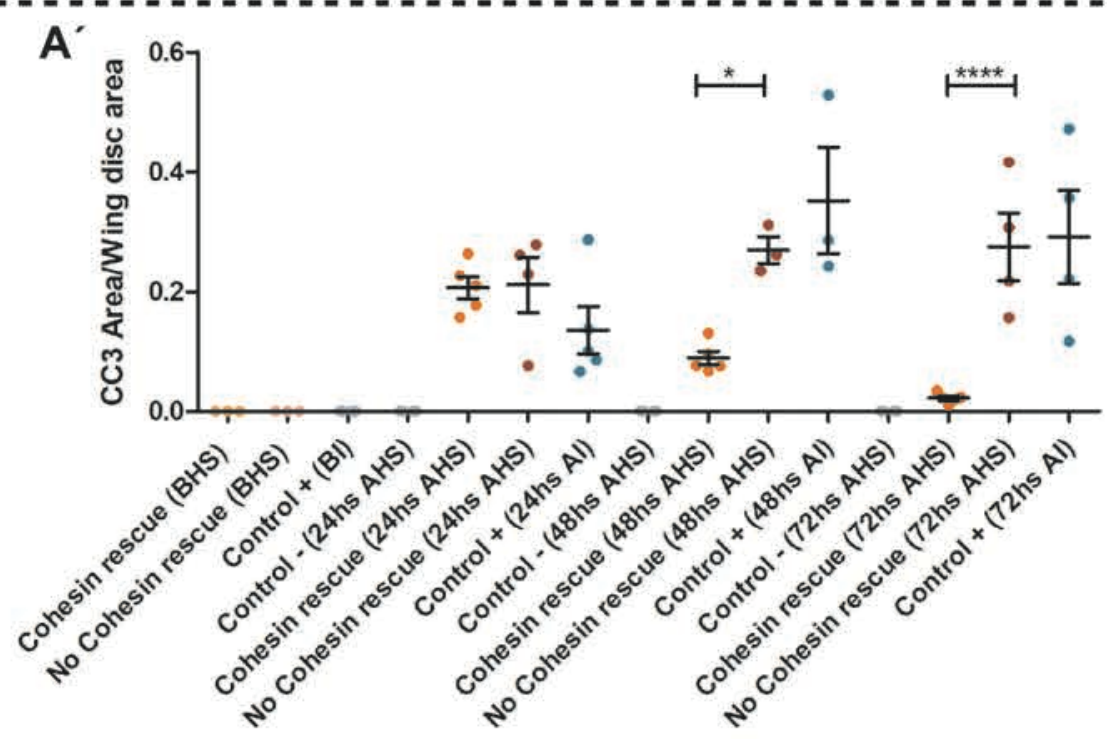




\section{Supp Figure 3}

A
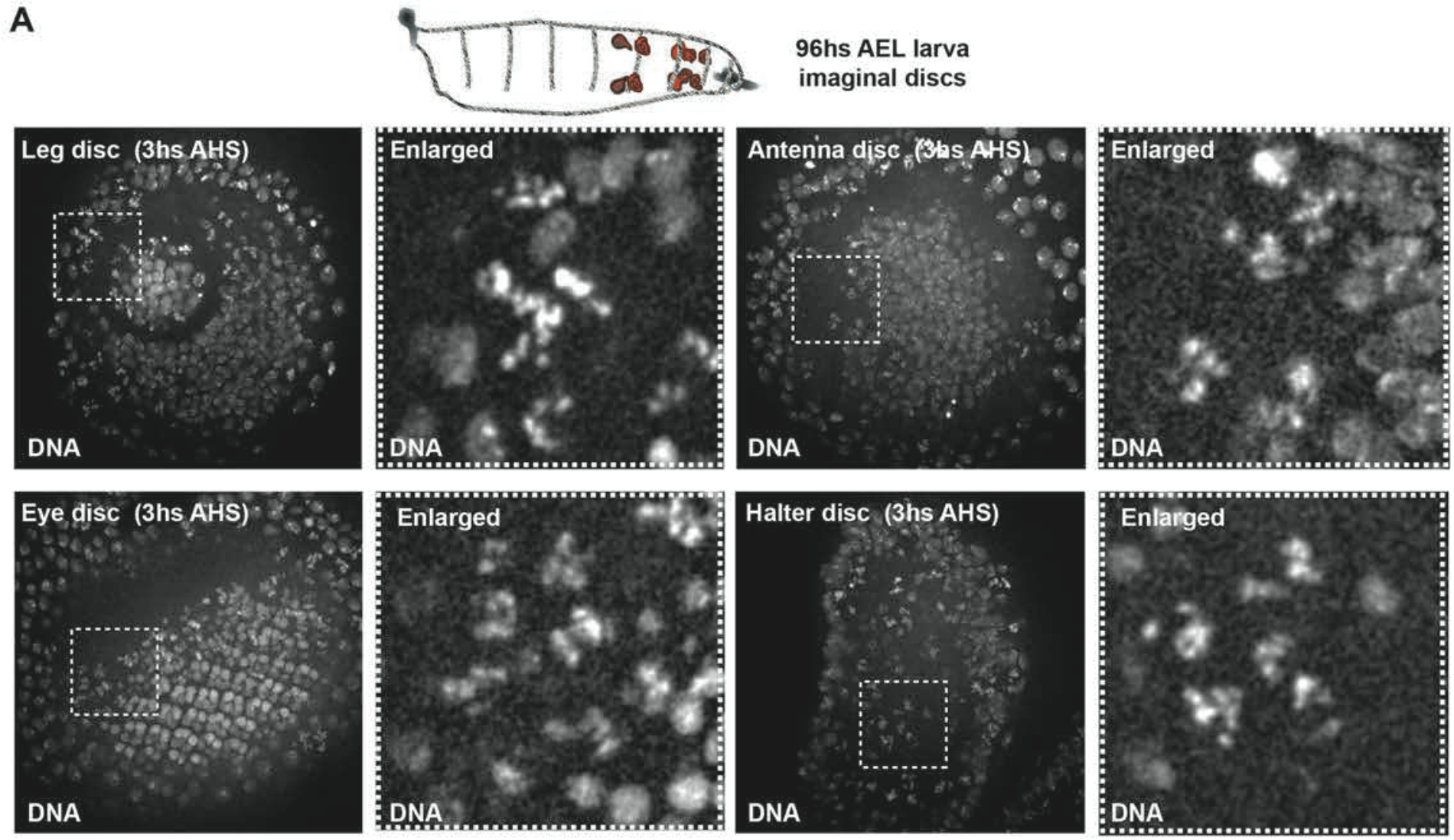

B

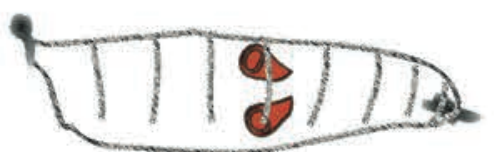

\section{6hs AEL larva} wing discs

\section{Before Heat-Shock}

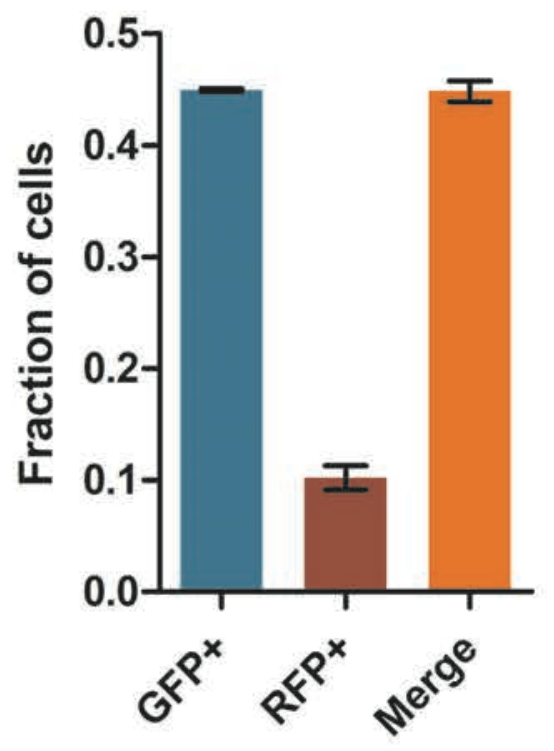

\section{After Heat-Shock}
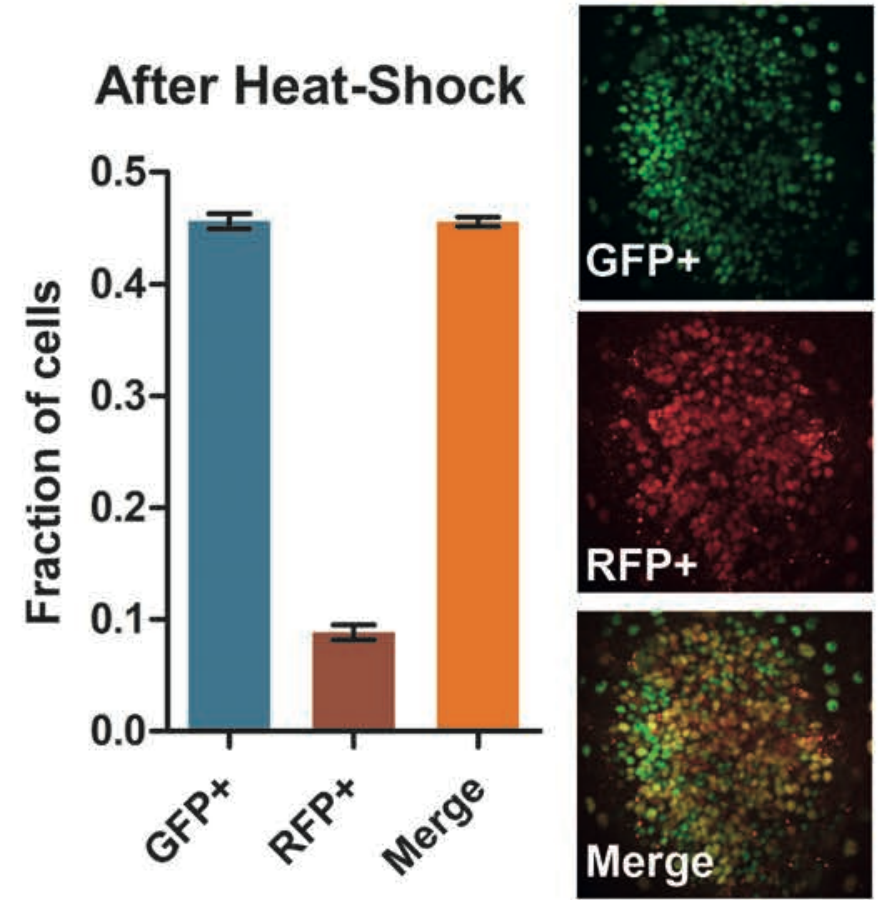
bioRxiv preprint doi: https://doi.org/10.1101/392746; this version posted August 15, 2018. The copyright holder for this preprint (which was

not certified by peer review) is the author/funder, who has granted bioRxiv a license to display the preprint in perpetuity. It is made available under aCC-BY 4.0 International license.

\section{Supp Figure 4}

A

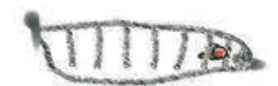

96hs AEL larva brains

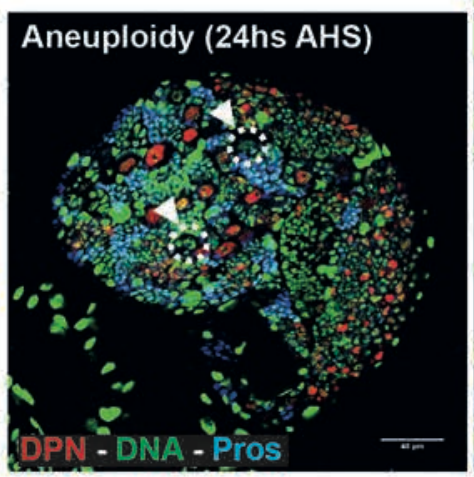

\section{B}
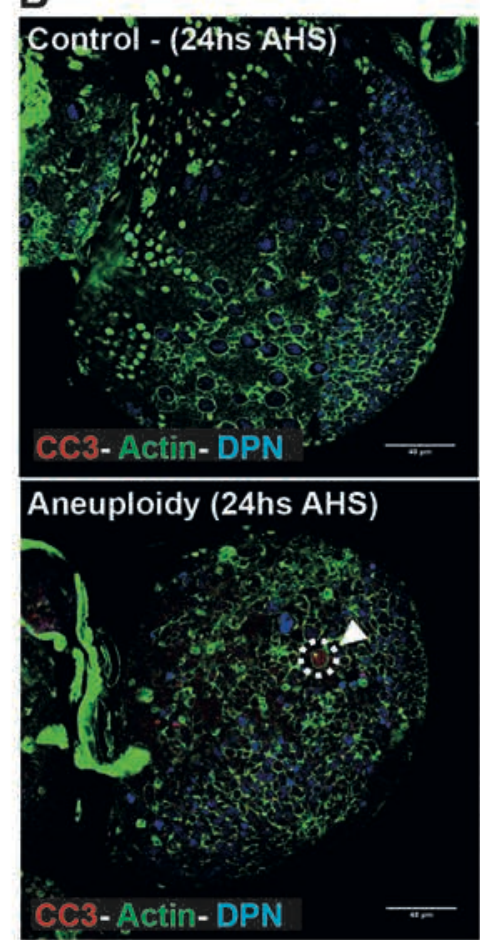

$B^{\prime}$

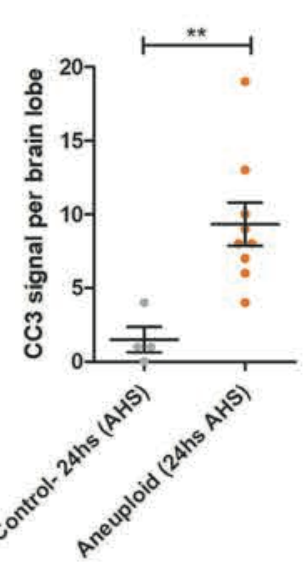

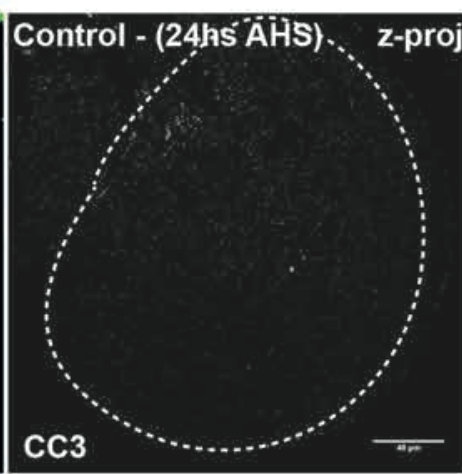

Aneuploidy (24hs AHS) z-proj

$\mathrm{CC}_{3}$

$\mathbf{B}^{\prime}$

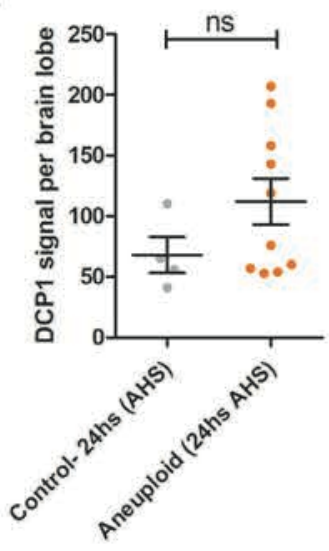

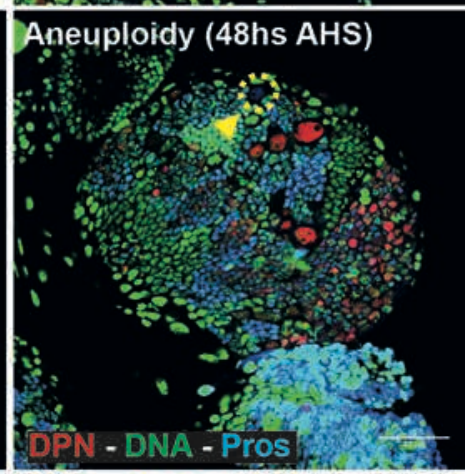
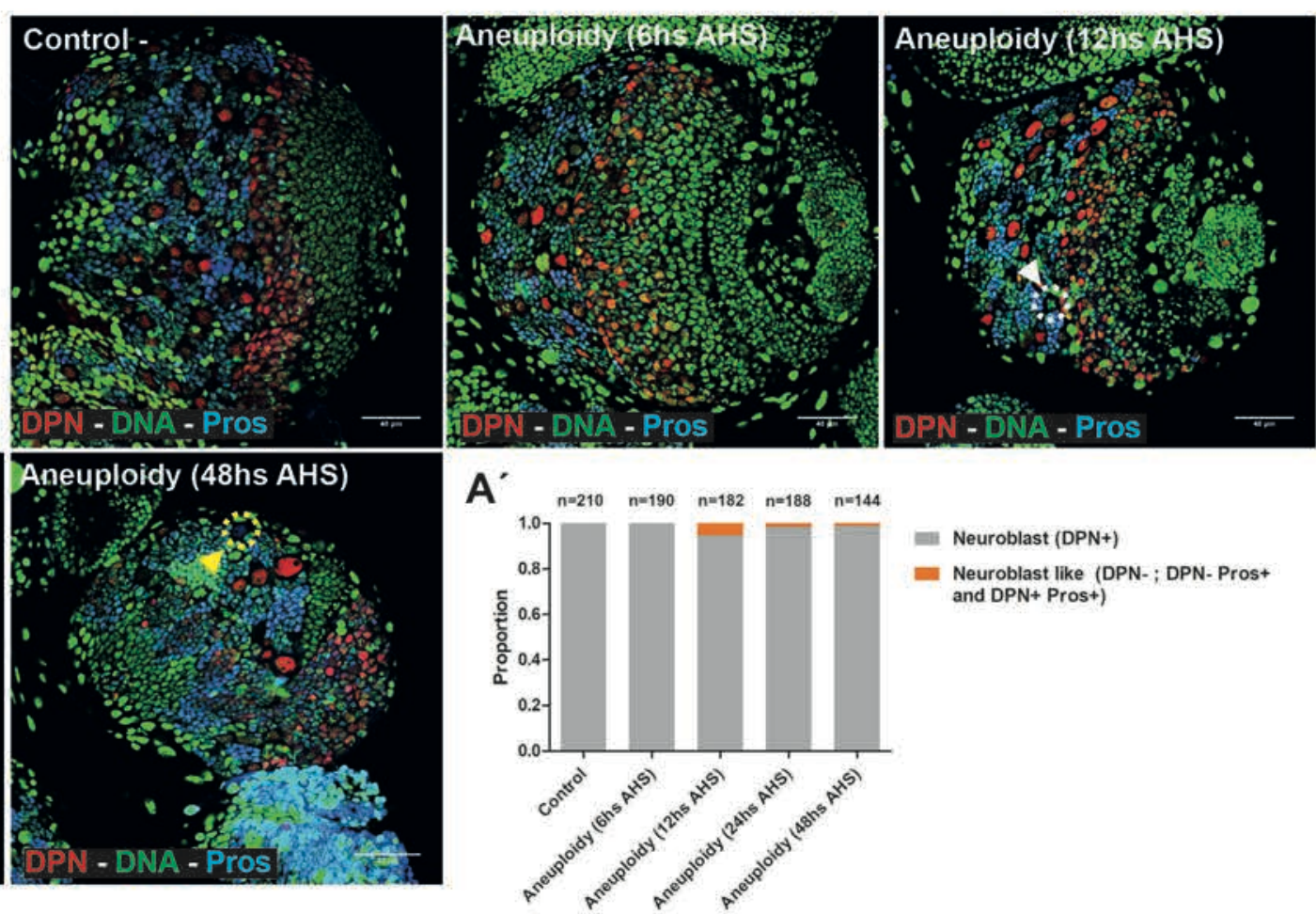

$$
\begin{aligned}
& \text { Neuroblast (DPN+) } \\
& =\begin{array}{l}
\text { Neuroblast like (DPN-; DPN-Pros+ } \\
\text { and DPN+ Pros+) }
\end{array}
\end{aligned}
$$
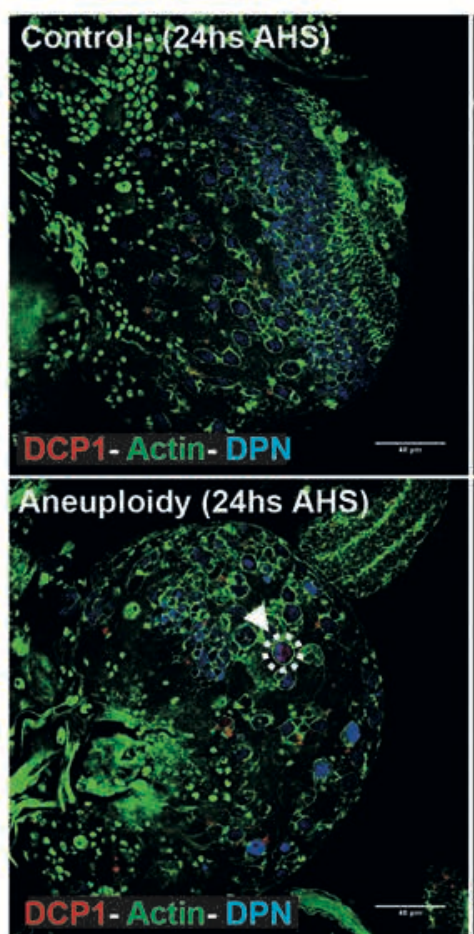

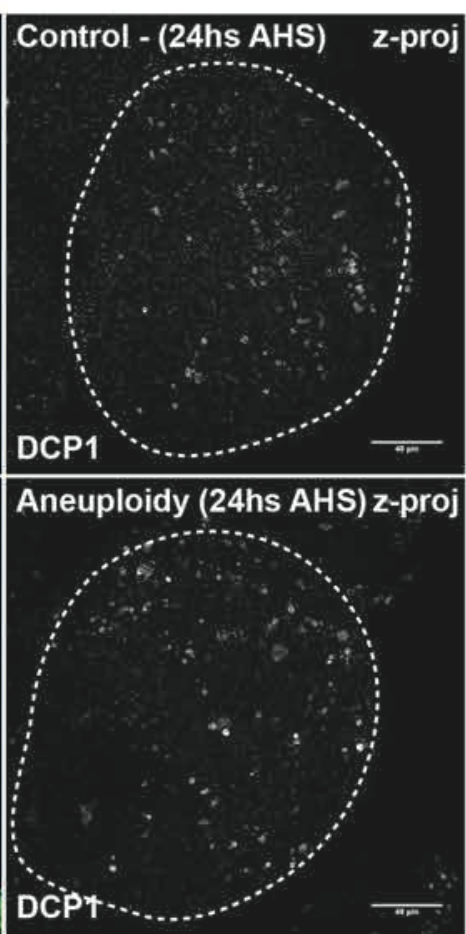

C

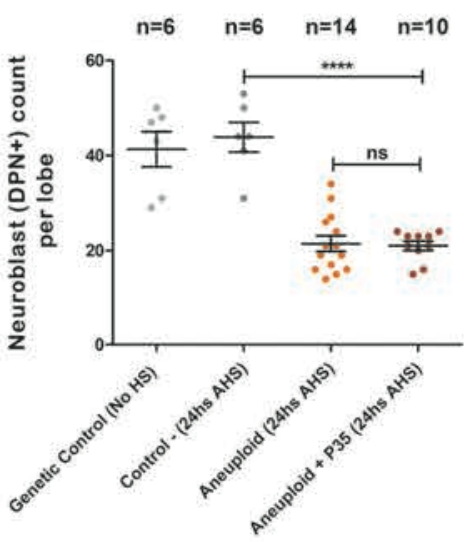


bioRxiv preprint doi: https://doi.org/10.1101/392746; this version posted August 15, 2018. The copyright holder for this preprint (which was

not certified by peer review) is the author/funder, who has granted bioRxiv a license to display the preprint in perpetuity. It is made available under aCC-BY 4.0 International license.

\section{Supp Figure 5}
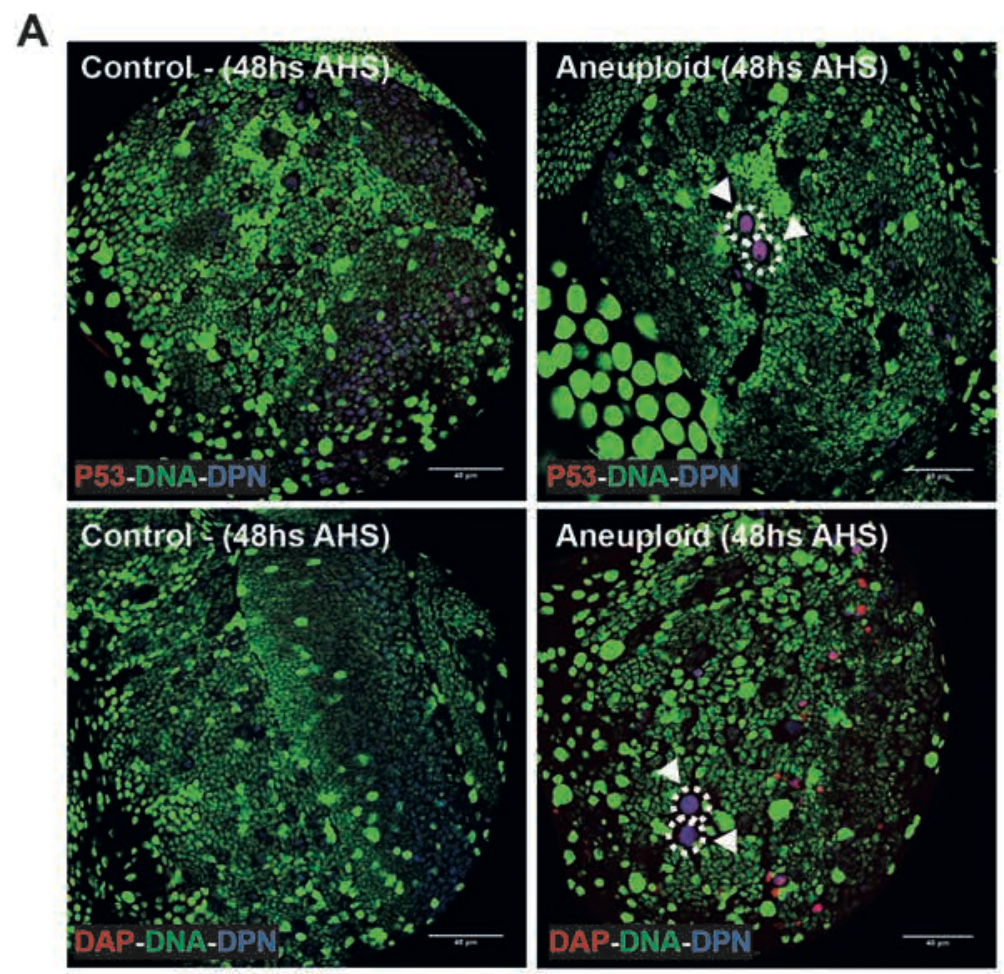

$A^{\prime}$
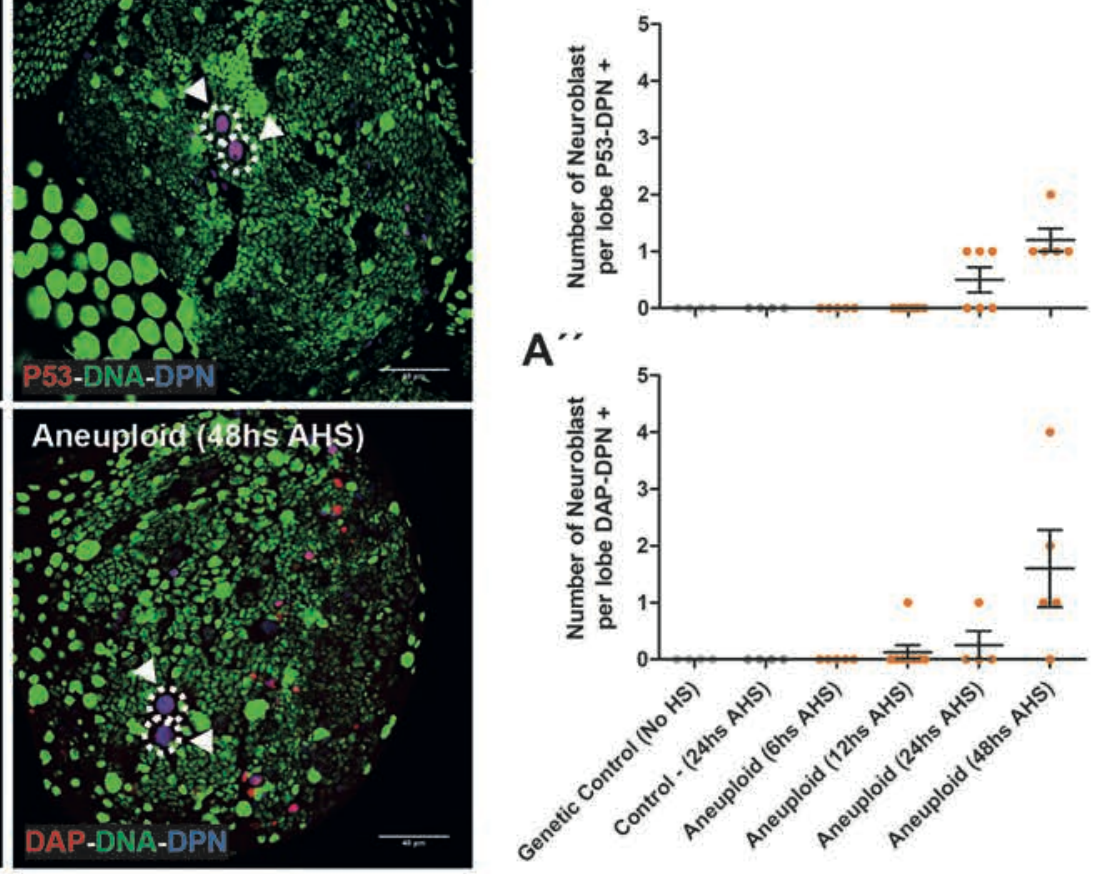
bioRxiv preprint doi: https://doi.org/10.1101/392746; this version posted August 15, 2018. The copyright holder for this preprint (which was

not certified by peer review) is the author/funder, who has granted bioRxiv a license to display the preprint in perpetuity. It is made available under aCC-BY 4.0 International license.

\section{Supp Figure 6}
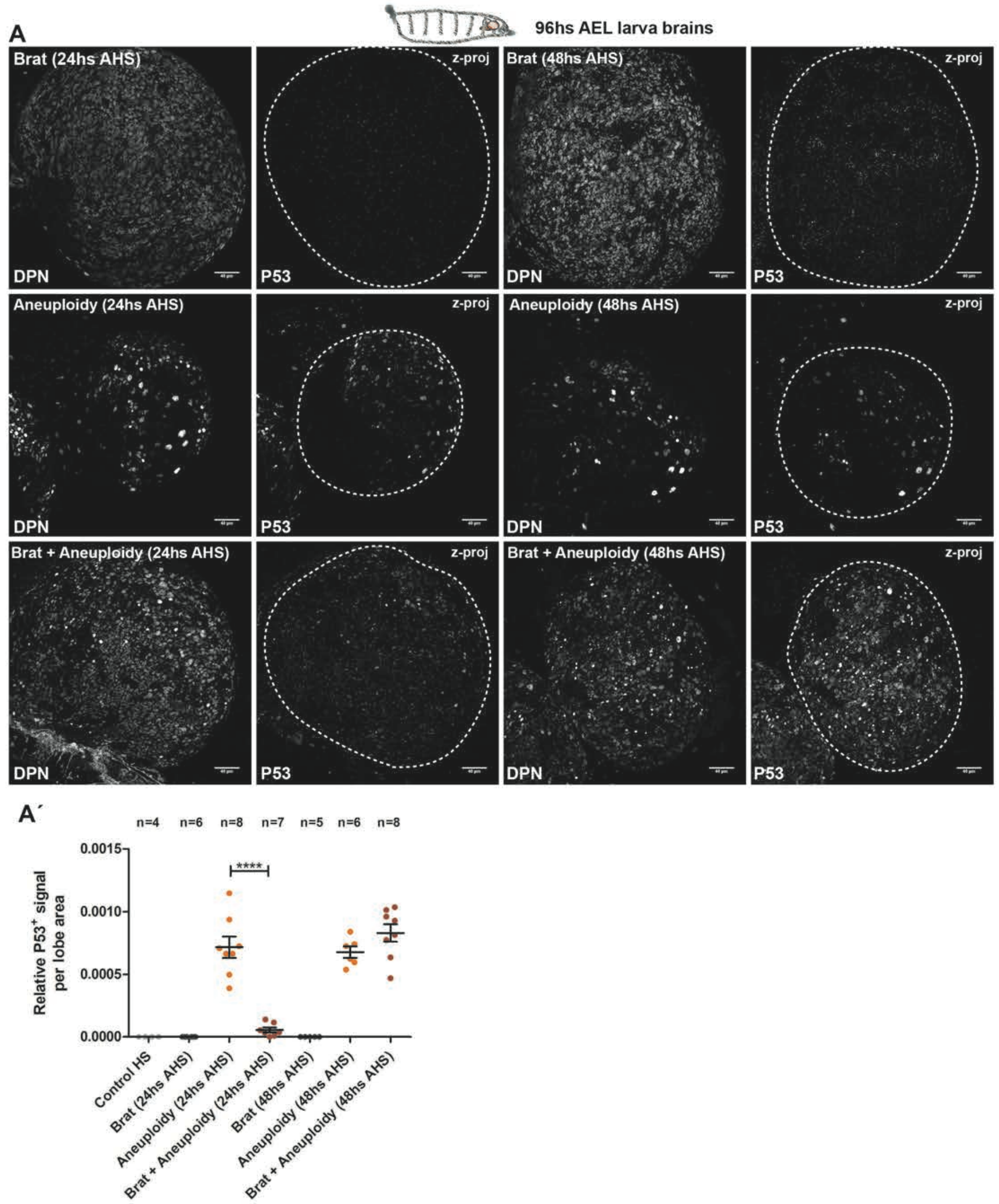


\section{Figure Supp 7}

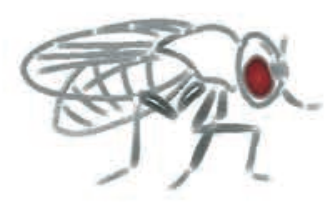

A Genetic Control (No HS)

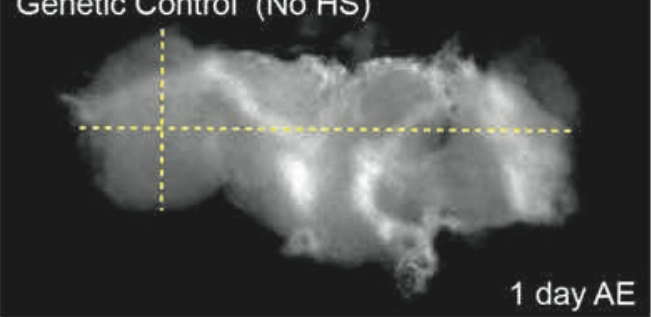

Aneuploidy induced (72hs AEL)

B

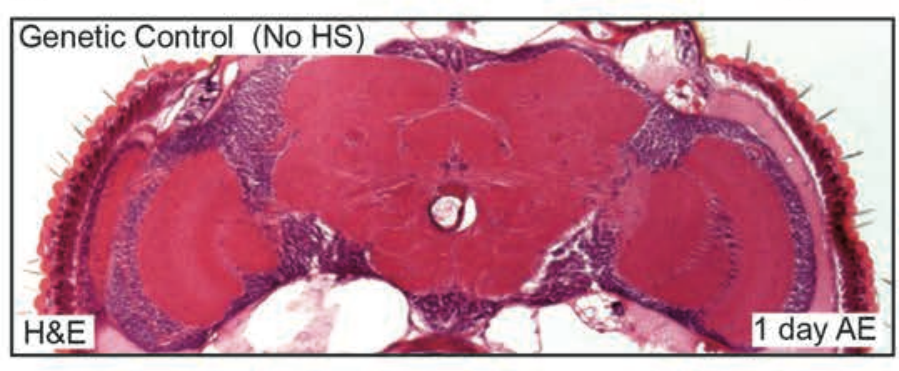

1 day $A E$
Brain length

$A^{\prime}$

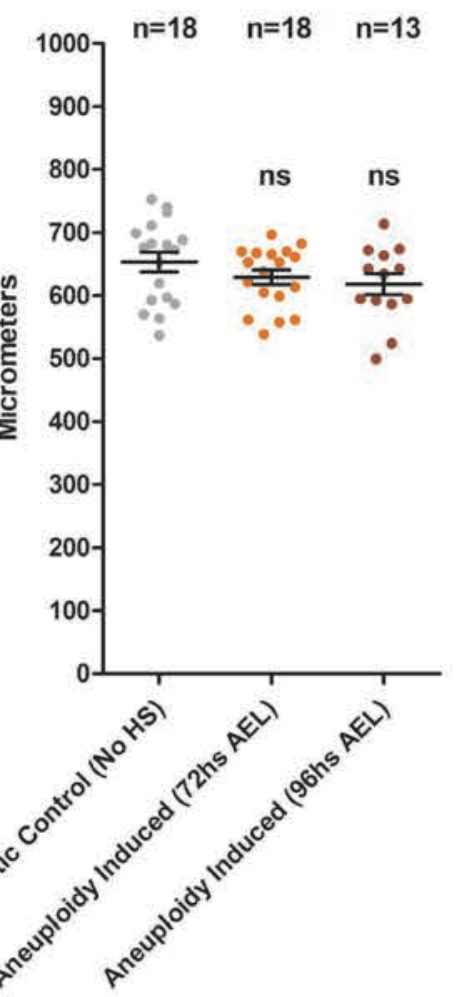

Lobe diameter

A
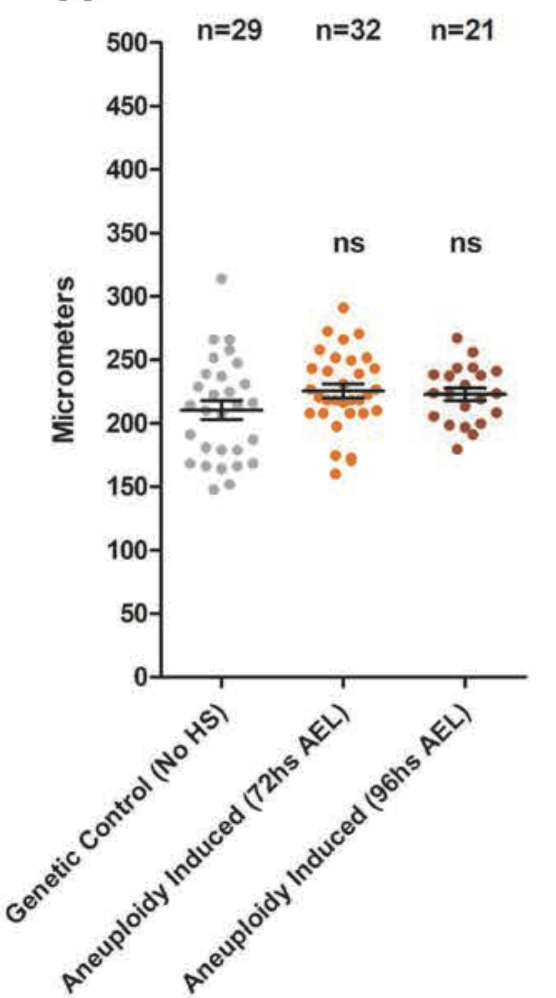

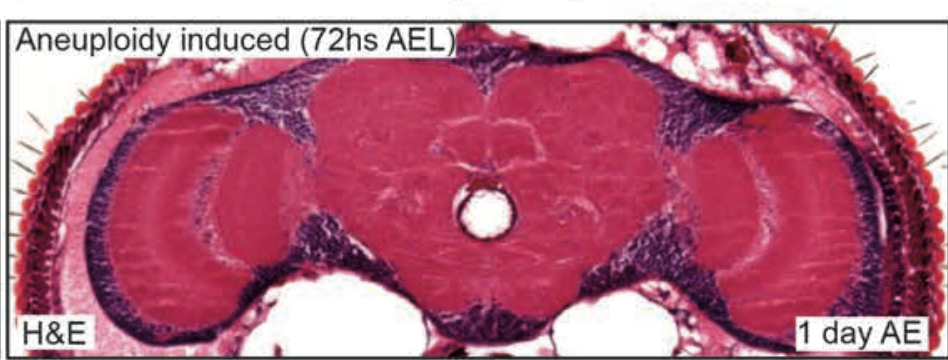

